\title{
LMC N132D: A mature supernova remnant with a power-law gamma-ray spectrum extending beyond $8 \mathrm{TeV}$
}

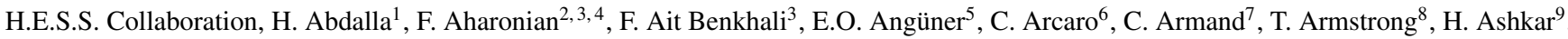

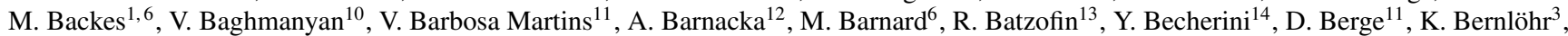

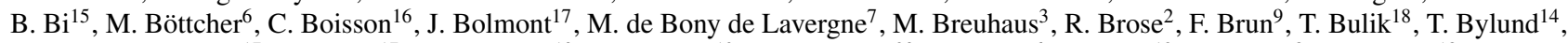

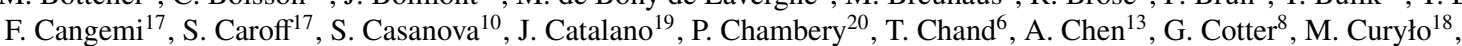

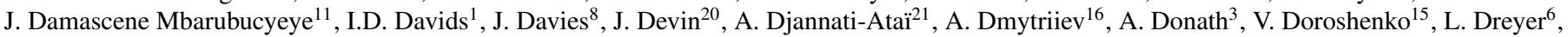

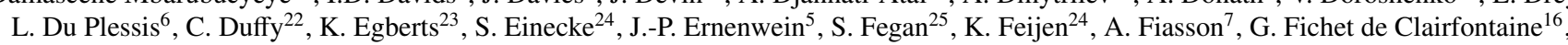

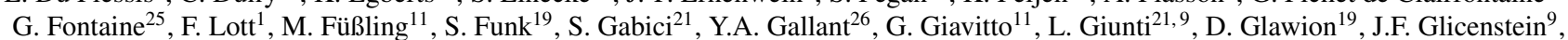

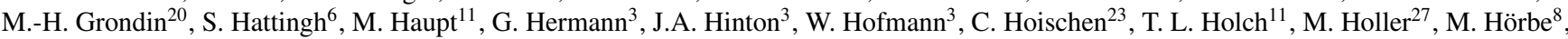

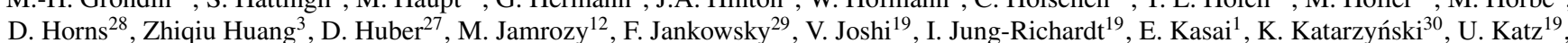

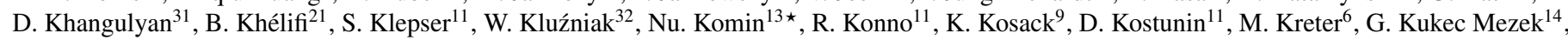

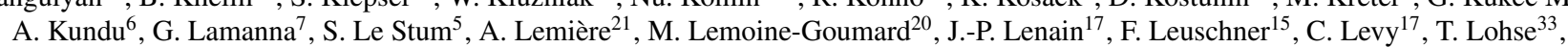

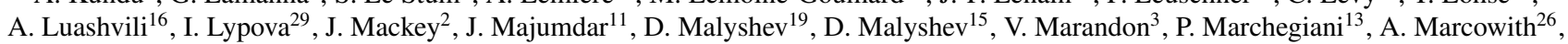

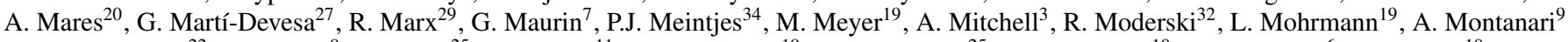

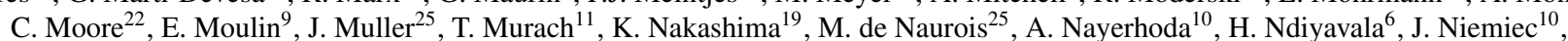

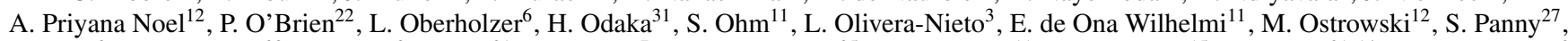

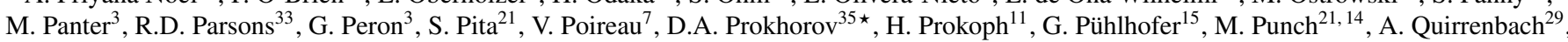

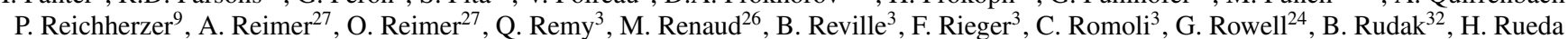

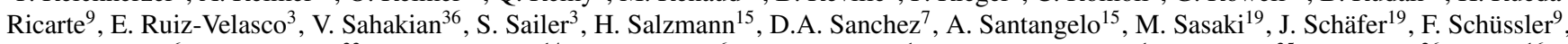

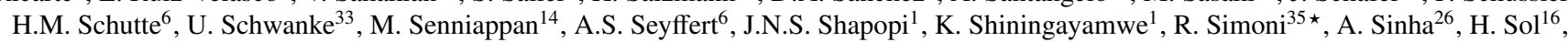

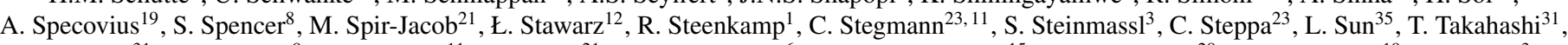

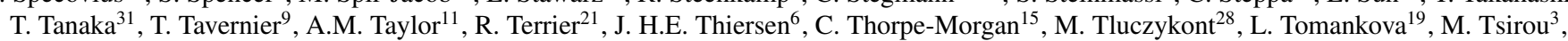

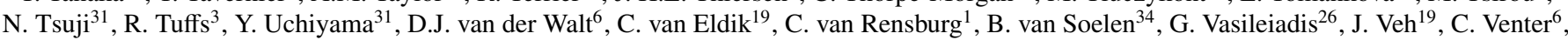

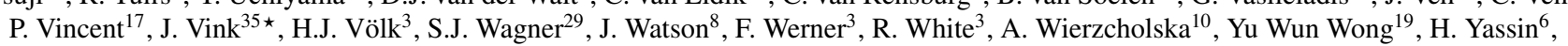

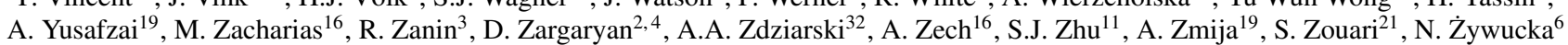

\section{(Affiliations can be found after the references)}

August 5, 2021

\section{ABSTRACT}

Context. Supernova remnants (SNRs) are commonly thought to be the dominant sources of Galactic cosmic rays up to the knee of the cosmic-ray spectrum at a few PeV. Imaging Atmospheric Cherenkov Telescopes have revealed young SNRs as very-high-energy (VHE, $>100 \mathrm{GeV}$ ) gammaray sources, but for only a few SNRs the hadronic cosmic-ray origin of their gamma-ray emission is indisputably established. In all these cases, the gamma-ray spectra exhibit a spectral cutoff at energies much below $100 \mathrm{TeV}$ and thus do not reach the PeVatron regime.

Aims. The aim of this work was to achieve a firm detection for the oxygen-rich SNR LMC N132D in the VHE gamma-ray domain with an extended set of data, and to clarify the spectral characteristics and the localization of the gamma-ray emission from this exceptionally powerful gamma-ray-emitting SNR.

Methods. We analyzed 252 hours of High Energy Stereoscopic System (H.E.S.S.) observations towardsSNR N132D that were accumulated between December 2004 and March 2016 during a deep survey of the Large Magellanic Cloud, adding 104 hours of observations to the previously published data set to ensure a $>5 \sigma$ detection. To broaden the gamma-ray spectral coverage required for modeling the spectral energy distribution, an analysis of Fermi-LAT Pass 8 data was also included.

Results. We unambiguously detect N132D at VHE with a significance of $5.7 \sigma$. We report the results of a detailed analysis of its spectrum and localization based on the extended H.E.S.S. data set. The joint analysis of the extended H.E.S.S and Fermi-LAT data results in a spectral energy distribution in the energy range from $1.7 \mathrm{GeV}$ to $14.8 \mathrm{TeV}$, which suggests a high luminosity of N132D at GeV and TeV energies. We set a lower limit on a gamma-ray cutoff energy of $8 \mathrm{TeV}$ with a confidence level of $95 \%$. The new gamma-ray spectrum as well as multiwavelength observations of N132D when compared to physical models suggests a hadronic origin of the VHE gamma-ray emission.

Conclusions. SNR N132D is a VHE gamma-ray source that shows a spectrum extending to the VHE domain without a spectral cutoff at a few $\mathrm{TeV}$, unlike the younger oxygen-rich SNR Cassiopeia A. The gamma-ray emission is best explained by a dominant hadronic component formed by diffusive shock acceleration. The gamma-ray properties of N132D may be affected by an interaction with a nearby molecular cloud that partially lies inside the $95 \%$ confidence region of the source position.

Key words. gamma rays: general, cosmic-ray: general, supernovae remnant: N132D

\section{Introduction}

Send offprint requests to: H.E.S.S. collaboration, e-mail: contact.hess@hess-experiment.eu;

^ Corresponding authors
Supernova remnants (SNRs) have long been thought to be the dominant sources of Galactic cosmic rays (CRs). If this is the 
case, then $5-10 \%$ of the explosion energy needs to be transferred to the CR particles to explain the CR energy budget (Ginzburg \& Syrovatskii 1964). This concerns atomic nuclei (hadronic CRs) in particular, which make up $\sim 99 \%$ of CRs that are detected on Earth. Gamma-ray observations provide a probe of energetic particles accelerated in SNRs. Accelerated electrons can produce gamma rays through their inverse Compton scattering and bremsstrahlung (the leptonic scenario), and accelerated hadrons can produce gamma rays through the production of short-lived neutral pions by proton-proton (or ion-ion) interactions (the hadronic scenario). In both cases, the gamma-ray photon energy is typically $\sim 10 \%$ of the energy of the accelerated particle. The highest CR energies to be expected in SNRs are at least $10^{14}-10^{15} \mathrm{eV}$ (Lagage \& Cesarsky 1983; Berezhko 1996), which should lead to gamma-ray emission in the TeV-sub $\mathrm{PeV}$ range.

To date, 25 sources associated with SNR 1 have been firmly detected in the very-high-energy gamma-ray regime (VHE, 100 $\mathrm{GeV}<\mathrm{E}<100 \mathrm{TeV}$, Abdalla et al. 2018c), some of which were even first discovered in VHE gamma rays (e.g., Abdalla et al.2018e). These VHE gamma-ray detections were achieved with ground-based Imaging Atmospheric Cherenkov Telescope (IACT) arrays. Thirteen of these sources have been identified as Galactic shell-type SNRs, ten additional sources are SNRs interacting with molecular clouds, and a few remaining sources are composite SNRs, that is, they also host a pulsar wind nebula (PWN).

In the high-energy regime ( $\mathrm{HE}, 100 \mathrm{MeV} \lesssim \mathrm{E} \lesssim 100 \mathrm{GeV}$ ), $\sim 30$ gamma-ray sources are classified as SNRs in the FermiLAT SNR catalog (Acero et al.2016). These include the mature SNRs ( 25000 yr) IC443 and W44, whose gamma-ray spectra display a clear pion bump signature, which provides evidence for a hadronic origin of the gamma-ray emission (Ackermann et al. 2013). The outer shocks of these two SNRs are interacting with dense molecular clouds, which act as reservoirs of target material for pion production. Their gamma-ray spectra show spectral breaks, suggesting that the highest-energy accelerated protons have largely escaped the SNR shell. Further evidence that VHE emission in mature SNRs originates from CRs that have escaped the SNR is provided by VHE gamma-ray observations of W28, for which VHE emission was detected from a nearby molecular cloud situated outside the shell of the SNR (e.g., Abramowski et al. 2008; Gabici 2017).

In general, gamma-ray studies show that (i) several young SNRs $(\lesssim 1000 \mathrm{yr})$ have prominent VHE gamma-ray emission, but there is no evidence that the underlying CR spectrum extends to the CR knee at $3 \times 10^{15} \mathrm{eV}$; the bright young SNR, Cassiopeia A (Cas A, $340 \mathrm{yr}$ ), exhibits a cutoff at a surprisingly low energy of 3.5 TeV in the gamma-ray spectrum (Ahnen et al.2017); (ii) mature SNRs evolving in dense regions are bright $\mathrm{GeV}$ sources, but exhibit a cutoff and/or break in their HE spectra, and they are therefore not prominent VHE gamma-ray sources; (iii) for some mature SNRs, it is suggested that the gamma-ray emission is caused by CRs that have escaped the SNR shell and are now colliding with dense nearby gas.

The SNR N132D is a remarkable object located in the Large Magellanic Cloud (LMC). At a distance of $\sim 50 \mathrm{kpc}$ (e.g., Pietrzyński et al. 2019), N132D is the only known extragalactic VHE emitting SNR (Abramowski et al.2015b). SNR N132D was discovered in gamma rays in the HE and the VHE regimes (Abramowski et al. 2015b, Ackermann et al. 2016). Its gammaray luminosity was estimated to be about $10^{30} \mathrm{erg} \mathrm{s}^{-1}$, making

${ }^{1}$ See http://tevcat.uchicago.edu/ it one of the most luminous VHE gamma-ray SNRs detected so far. It is the brightest X-ray SNR in the LMC of 38 cataloged LMC SNRs (Maggi et al. 2016). However, unlike many other VHE-emitting shell-type SNRs, N132D shows only strong thermal X-ray emission (e.g., Mathewson et al. 1983; Hughes et al. 1998; Behar et al. 2001; Borkowski et al. 2007) without evidence for an X-ray synchrotron component (e.g., Bamba et al. 2018). It is also a luminous source in the radio (Dickel \& Milne 1995) and infrared (IR) bands (Seok et al. 2013). There is evidence that N132D was caused by a powerful explosion with an energy of (3-5) $\times 10^{51}$ erg (Dickel \& Milne 1995; Hughes et al. 1998), three to five times more than the canonical supernova explosion energy.

N132D has a peculiar horseshoe morphology in the X-ray band (see, e.g., Fig. 2 in Borkowski et al.2007) and also in the radio and IR bands, with a typical angular size of $1.4^{\prime}$ by $1.8^{\prime}$. This corresponds to a physical size of about $20 \mathrm{pc}$ by $26 \mathrm{pc}$. The northeast region of the SNR is fainter than the southwest region, which suggests that the SNR interacts with denser material in the southwest. Molecular clouds are projected toward the southwestern region of the remnant (e.g., Braiding et al. 2018). $\mathrm{HI}$ emission also shows that N132D is surrounded by relatively dense HI gas, with a gradient toward the southwest. Sano et al. (2020) estimated an HI density as high as $n \approx 30 \mathrm{~cm}^{-3}$. It seems plausible that in the southern part of the shell, molecular cloud material has been swept up and heated by a high-speed shock, resulting in the peculiar morphology of N132D (see Williams et al. 2006; Tappe et al. 2007, 2012). Sano et al. (2020) also reported that there are small clouds in the south and in the center of N132D that shows signs of present or past interaction with the shock.

The age of N132D is estimated to be $\sim 2500$ years (Vogt \& Dopita 2011). It is therefore one of the oldest VHE gamma-rayemitting shell-type SNRs. It is much older than Cas A ( 340 yr), to which it is often compared in other aspects: both SNRs probably have evolved in a wind-blown bubble, and both contain oxygen-rich ejecta, which suggest that their explosions were the result of core-collapse supernovae of massive stars $\left(\gtrsim 18 \mathrm{M}_{\odot}\right.$, at the zero-age main sequence). The age of another oxygen-rich SNR, Puppis A, is similar to that of N132D, but it has not yet been detected in VHE gamma rays although it is located only $1.3 \mathrm{kpc}$ away (Abramowski et al.2015a). The difference in VHE gamma-ray properties of N132D, Cas A, and Puppis A makes a detailed study of N132D relevant for the important question of the timescale on which the bulk of CRs are accelerated, and of the timescale on which the CRs eventually escape the shells as a function of CR energy.

Abramowski et al. (2015b) reported a gamma-ray excess from N132D at a statistical significance level of $4.7 \sigma$ and a spectrum extending up to $10 \mathrm{TeV}$, based on H.E.S.S. observations with an exposure time of 148 hours. Our goal is to update this result by reporting the analysis of H.E.S.S. observations with 252 hours of total exposure time. The HE gamma-ray counterpart of VHE emission is also studied with Fermi-LAT Pass 8 data. This joint study in the HE and VHE gamma-ray bands allows us to place new constraints on the mechanism of gamma-ray production and the underlying populations of accelerated particles responsible for the gamma-ray emission.

The content of this paper is structured as follows: In section 2 the data analysis of both H.E.S.S. and Fermi-LAT observations are described. In Section 3 the H.E.S.S VHE spectrum and gamma-ray morphology are presented. In Section 4 a multiwavelength spectrum modeling is explained. These results are discussed in Section 5 in the context of the environment of N132D 
as well as in the context of the VHE-emitting SNR population. The conclusions of this study are presented in Section 6

\section{Observations and data analysis}

\subsection{H.E.S.S. experiment}

The High Energy Stereoscopic System ${ }^{2}$ (H.E.S.S.) is an array of five IACTs located in the Khomas Highland in Namibia at an altitude of $1800 \mathrm{~m}$ above sea level. It has been operating since December 2003. In its first phase ("H.E.S.S. I"), the array consisted of four identical 12m Cherenkov telescopes (CT1-4) placed at the corners of a square of $120 \mathrm{~m}$ side length. CT1-4 are equipped with mirrors with a total area of $107 \mathrm{~m}^{2}$ and cameras with 960 photo-multiplier tubes each. The stereoscopic IACT technique allows determining the energy and direction of VHE gamma rays through imprints of the Cherenkov light emitted by secondary charged particles of a shower initiated by a primary VHE photon entering the atmosphere. With the $5^{\circ}$ field of view (FoV) of CT1-4, gamma rays can be reconstructed with a typical angular resolution of $\sim 0.1$ ( $68 \%$ containment radius). The energy threshold is $\sim 100 \mathrm{GeV}$ for small zenith angles and below $1 \mathrm{TeV}$ for zenith angles up to $60^{\circ}$, and the energy resolution is $\sim 15 \%$ (for further details, see Aharonian et al. 2006). The fifth Cherenkov telescope (CT5) with a diameter of $28 \mathrm{~m}$ (mirror area of $614 \mathrm{~m}^{2}$ ) was added in the center of the CT1-4 array in July 2012, initiating the second phase of the experiment, "H.E.S.S. II". This study mostly uses H.E.S.S. I observations because only about $5 \%$ of the data were taken by the full CT1-5 array. H.E.S.S. performance depends on the zenith angle, configuration cuts, and the number of telescopes considered in the reconstruction (for more details, see sections 2.2 and 2.3.

\subsection{Data set: LMC survey}

Its location in the southern hemisphere makes H.E.S.S. the only currently operating IACT array able to observe the LMC in the VHE band. With its angular size of $\sim 8^{\circ}$, the LMC galaxy is compact enough for the H.E.S.S. telescopes to perform a survey. Such a survey was conducted over 13 years, from 2004 to 2017, during which the full LMC galaxy was covered with over 325 hours of observations. The exposure of the LMC is rather inhomogeneous and mostly concentrated on the central region around the Tarantula nebula. H.E.S.S. observations from 2003 to 2012 led to the discovery of three individual VHE gamma-rayemitting sources, including SNR N132D, along with the PWN of N 157B and the superbubble 30 Dor C (Abramowski et al. 2012, 2015b), as well as the detection of the gamma-ray binary LMC P3 (Abdalla et al.2018f). The data set used for our analysis was taken between December 2004 and March 2016. It consists of 252 hours of total exposure time, adding 104 hours to the previously published data set. The zenith angle of these observations spans a range of $45-57^{\circ}$, with a mean of $46^{\circ}$, and a mean azimuth angle of 182.5 . The data set was selected with a maximum offset of 2.5 around the source to avoid observations with large offset angles with respect to the camera center. For N132D, the observation positions were mostly toward the east side of the SNR. This is due to the nature of the observations, which are part of a survey, instead of being dedicated to N132D: the pointings were mostly directed toward N157B in the Tarantula nebula, which is situated toward the east of N132D. This results in offset angles in the range of 0.16 to 2.5 and a mean offset angle for N132D of

\footnotetext{
2 https://www.mpi-hd.mpg.de/hfm/HESS/
}

1.13. The observations are thus taken at rather large offset, for which the acceptance is reduced compared to sources located closer to the camera center. Observations at large zenith angles with IACTs in general result in a lower effective area at energies below $1 \mathrm{TeV}$, but an increase in effective area above $1 \mathrm{TeV}$ (e.g., Acciari et al. 2020). Therefore these observations were particularly well suited for measuring the high-energy part of the N132D gamma-ray spectrum.

\subsection{H.E.S.S. data analysis}

Data corresponding to 252 hours were recorded in 28-minute exposures that are called runs. Each calibrated run that passed the quality criteria of a dead time shorter than $30 \%$ of the run duration, a low fluctuation in trigger rate, and acceptable weather conditions (see Aharonian et al. 2006; Hahn et al. 2014) was analyzed using the ImPACT framework described by Parsons \& Hinton (2014), which includes a boosted decision-tree-based event classification algorithm to distinguish gamma rays from the charged particle background (Ohm et al. 2009). This analysis was performed using a special configuration that selected events with a minimum of three shower images in different telescopes. Using this event selection cut allowed us to improve the signal-to-background ratio for this faint gamma-ray source in the presence of a strong diffuse component, or multiple unresolved sources constituting an astrophysical background, which may be present in the vicinity of N132D.

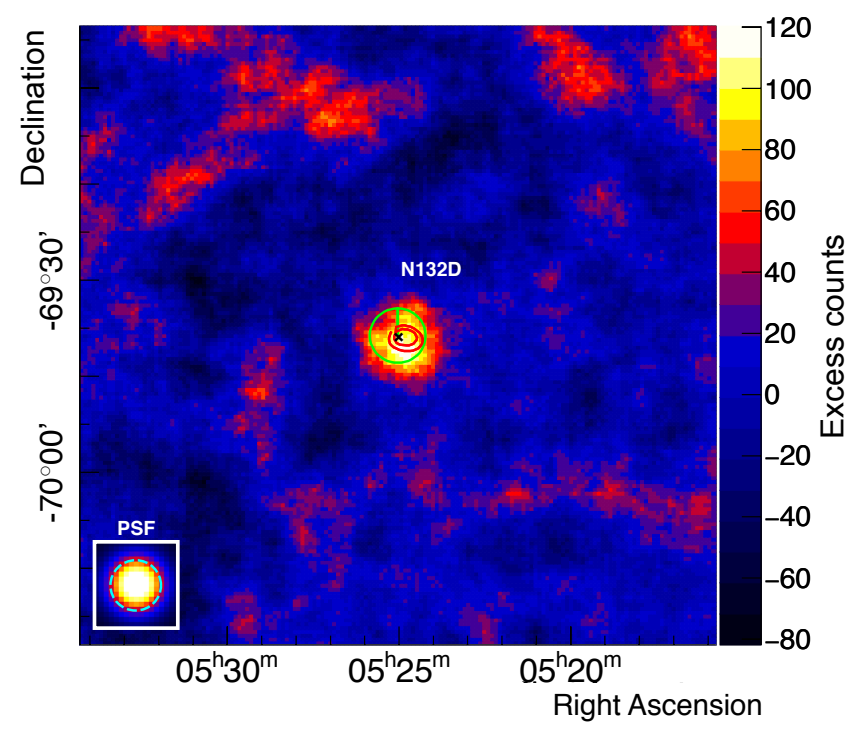

Fig. 1. H.E.S.S. excess map with a size of 1.6 , smoothed with a Gaussian of width $0: 1$. The $\mathrm{ON}$ region with a radius of $0: 07$, centered on the N132D X-ray source position, is shown in green, and the 2 and $3 \sigma$ of the best-fit position (see section 3.2 ) are represented in red.

To compute the maps and statistics presented in section 3 $\mathrm{ON}$ events were selected from a circle centered on the geometrical center of the X-ray emission (referred to as the "X-ray source position") at RA $5^{\mathrm{h}} 25^{\mathrm{m}} 2.2^{\mathrm{s}}$, Dec $-69^{\circ} 38^{\prime} 39^{\prime \prime}$. OFF events were selected according to a ring-background method (Berge et al. 2007). An additional cut on the energy of reconstructed gammarays was made above $1.3 \mathrm{TeV}$ in order to improve the signal-tobackground ratio for this hard gamma-ray source and keep only gamma-ray events from a well-determined direction because the angular resolution improves by $10 \%$ from $800 \mathrm{GeV}$ to $1.3 \mathrm{TeV}$ using the ImPACT shower reconstruction software. This resulted 
in a point spread function (PSF) of 0.06 , which corresponds to the $68 \%$ containment radius of the fit on the observation position. The event count excess was computed using $\mathrm{N}_{\text {excess }}=\mathrm{N}_{\text {on }}$ $\alpha \mathrm{N}_{o f f}$, with $\alpha$ the normalization factor for the OFF area with respect to the $\mathrm{ON}$ area. The cumulative statistical significance was calculated using Equation 17 of $\mathrm{Li} \& \mathrm{Ma}(1983)$. The excess map is shown in Fig. 1 .

For the spectrum, the reflected-background method was applied with a three-telescope-image selection cut and a lowenergy cut above $1.3 \mathrm{TeV}$. The spectral background was derived from background control regions that were defined run-wise and were chosen to have the same offset to the camera center as the source region to ensure a nearly identical spectral response. For maps and the spectrum, the sky areas with known sources or with excess events above a certain significance threshold were excluded. For reliability, the results were cross-checked with an independent analysis and calibration chain (de Naurois \& Rolland 2009).

\subsection{Fermi-LAT data analysis}

The Large Area Telescope (Fermi-LAT) is a high-energy gamma-ray telescope installed on the Fermi spacecraft (Atwood et al. 2009). It uses a pair-conversion technique for gamma-ray photon detection, and has a large FoV of about $20 \%$ of the sky. The Fermi-LAT has been scanning the entire sky since August 2008, recording astrophysical gamma rays with energies in the range $30 \mathrm{MeV}$ to $300 \mathrm{GeV}$. Despite the uniform coverage of the entire LMC galaxy and the excellent spectral capabilities of Fermi-LAT, the previous analyses of Fermi-LAT observations were inconclusive regarding the origin of gamma-ray emission from N132D. In particular, different values of the photon spectral index were reported: $\Gamma=1.4 \pm 0.3$ in Ackermann et al. (2016) and $\Gamma=2.07 \pm 0.19$ in the LAT 8-year source catalog (the 4FGL catalog; Abdollahi et al. 2020). In the latter work, the source 4FGL J0524.8-6938 was identified with N132D, and it was argued that the derived photon index of 2.07 supports the hypothesis of dominantly hadronic emission, while for SNRs exhibiting gamma-ray spectra with very hard photon indices, $\Gamma \leqslant 1.6$, the gamma-ray emission is most likely of leptonic origin (Abdollahi et al. 2020).

Pass 8 R3 SOURCE class photon dat $3^{3}$ (evclass $\left.=128\right)$ spanning 10.8 years between August 4, 2008, and May 15, 2019 , with energies between $1 \mathrm{GeV}$ and $250 \mathrm{GeV}$ were selected. For the data analysis, the FERMITOOLS v1.0.7 package and P8R3_SOURCE_V2 instrument response functions were used. Contamination from the gamma-ray-bright Earth limb was avoided by removing all events with zenith angle larger than $90^{\circ}$. The recommended quality cuts (DATA_QUAL $>0$ \& \& LAT_CONFIG $==1$ ) were applied. The $10^{\circ} \times 10^{\circ}$ square region of interest with spatial bins 0.05 in size was centered on the position of N132D. A Fermi-LAT count map in the range 3$250 \mathrm{GeV}$ smoothed with a Gaussian kernel is shown in Fig. 2 . The choice of the low-energy limit at $3 \mathrm{GeV}$ was made for illustration purposes because it provides a narrower point spread function. The central region of $1.6 \times 1.6$ in Fig. 2 indicated with the dashed line corresponds to the H.E.S.S. excess map shown in Fig. 1. Given the diameter of $\sim 1.6^{\prime}$ of N132D in X-rays, a point-like source model for its counterpart in HE gamma rays was adopted. Gamma-ray sources from the 4FGL catalog within a $17^{\circ}$ radius from $\mathrm{N} 132 \mathrm{D}$ were included to model the data. To model the diffuse emission from the LMC, the spatial templates

\footnotetext{
3 https://fermi.gsfc.nasa.gov/ssc/data/
}

for four diffuse sources, LMC-FarWest, LMC-Galaxy, LMC30DorWest, and LMC-North, provided by the Fermi-LAT collaboration (Ackermann et al. 2016), were used. These sources are outlined with circles in Fig. 22 To model the Galactic and isotropic background diffuse emission, the standard templates gl1_iem_vQ7.fits and iso_P8R3_SOURCE_V2_v1.txt were used. The spectral shapes of the sources were taken from the 4FGL catalog. The normalization and the photon index of N132D were derived from the likelihood analysis along with the normalizations of four strong point sources, including 4FGL J0537.8-6909 and 4FGL J0540.3-6920, and all diffuse sources, while the normalizations of fainter point sources were held fixed at the 4FGL catalog values. A binned likelihood analysis was applied to the data using the Fermi Science tool gtlike. To assess the goodness of fit, test-statistic and residual maps were produced showing some residuals at the position of SNR N63A. However, the inclusion of a new source corresponding to N63A in the model negligibly affects the derived spectrum of N132D. To extract a spectral energy distribution (SED), the photon index of N132D derived to be $1.86 \pm 0.25$ was fixed at the central value, the data were rebinned in four broad logarithmically spaced bands in energy, and a binned likelihood analysis was applied to the data in each of these bands.

The main analysis with all LMC templates was first performed, providing the best estimate of the SNR N132D HE emission. A second analysis was then launched to estimate the uncertainties related to the presence of a molecular cloud near N132D. For this purpose, LMC-Galaxy and LMC-30DorWest templates were modified by patching the area enclosing the molecular cloud, with constant intensities matching those in the nearby region and keeping their initial spectral shapes. Given the proximity of N132D and the molecular cloud, the alternative model predicts a stronger HE gamma-ray signal from N132D and leads to an expected VHE flux that is higher by about $30 \%$, which is an estimate of the HE emission of SNR N132D plus the nearby molecular cloud. The data points obtained from the Fermi-LAT analysis are shown in Fig. 3 for the main model.

\section{Results}

\subsection{Statistics and spectral properties}

The H.E.S.S analysis of N132D reveals an excess of 95 gammaray events above an energy of $1.3 \mathrm{TeV}$, corresponding to a significance of $5.7 \sigma$ for an exposure of 252 hours. The VHE gammaray emission from N132D is thus unambiguously detected, for which there was previous evidence from H.E.S.S. at a lower significance level of $4.7 \sigma$ (Abramowski et al.2015b).

The excess corresponds to $337 \mathrm{ON}$ events and $11179 \mathrm{OFF}$ events, and a normalization parameter $\alpha=0.0216$ established with the ring-background method with standard settings. ON events were extracted in a circle of radius 0.07 centered on the $\mathrm{X}$-ray source position. The corresponding excess map is shown in Fig. 1 .

Fig. 3 shows the combined H.E.S.S. and Fermi-LAT photon energy spectrum spanning an energy range of $1 \mathrm{GeV}$ to $40 \mathrm{TeV}$, where H.E.S.S. data points were chosen to start at $1.3 \mathrm{TeV}$. The H.E.S.S. spectrum was extracted using the same analysis cuts as for the maps and the reflected-background method. Spectral points between 1.3 and $40 \mathrm{TeV}$ were binned in energy bands requiring a minimum of $2 \sigma$ significance per point. The H.E.S.S. spectrum exhibits a $2 \sigma$ significant spectral bin at $\sim 15 \mathrm{TeV}$.

The H.E.S.S. spectrum is well fit with a single power law $\left(d N / d E=\Phi_{0} \times(E / 1 \mathrm{TeV})^{-\Gamma}\right)$ with an index $\Gamma=2.3 \pm 0.2$ and a 


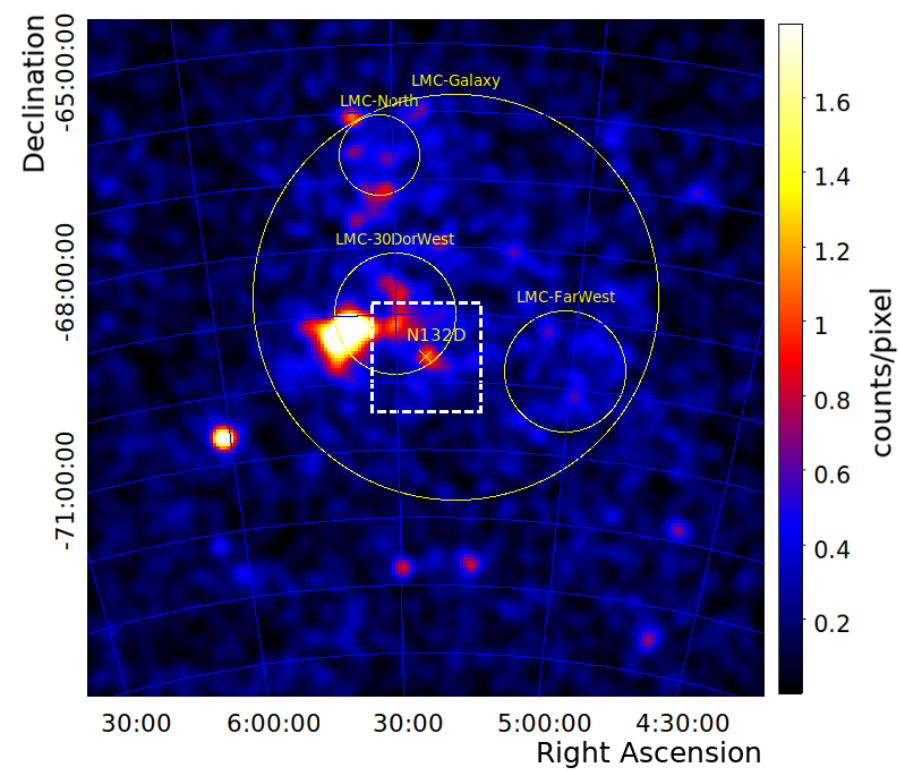

Fig. 2. Gaussian $(\sigma=0.2)$ kernel smoothed Fermi-LAT count map centered on the position of N132D for the energy range 3-250 GeV. The central dashed box corresponds to the FoV of the H.E.S.S. excess map shown in Fig. 1 The bright Fermi-LAT sources at the eastern edge of the LMC-30 DorWest region are N157B and PSR B0540-69. Their emissions overlap at this resolution.

differential flux normalization at $1 \mathrm{TeV}$ of $\Phi_{0}=(1.31 \pm 0.43) \times$ $10^{-13} \mathrm{TeV}^{-1} \mathrm{~cm}^{-2} \mathrm{~s}^{-1}$, with a $\chi^{2}$ of 3.9 for 2 degrees of freedom. The derived photon index is consistent with the previous estimate by H.E.S.S., yielding $\Gamma=2.4 \pm 0.3$ (Abramowski et al. $2015 b$ ). Systematic errors on the spectrum parameters are estimated to be \pm 0.3 for $\Gamma$, and $\pm 30 \%$ for $\Phi_{0}$. These systematic uncertainties arise because the data set spans 13 years and the LMC is mostly observed during the rainy season, leading to significant variations in atmospheric conditions (see Abramowski et al. 2012). The corresponding luminosity, assuming a distance $d=50 \mathrm{kpc}$ to the source, is $L(1-10 \mathrm{TeV})=(1.05 \pm 0.29) \times$ $10^{35}\left(d / 50 \mathrm{kpc}^{2} \mathrm{erg} \mathrm{s}^{-1}\right.$. This value is consistent with previous results and is comparable to the luminosity of the brightest SNRs in the VHE band: N132D is as luminous as HESS J1640-465 (Abramowski et al. 2014) and 28 times more luminous than Cas A (Aharonian et al. 2001).

A combination of the H.E.S.S and Fermi-LAT data allowed us to investigate the spectral properties in a wide energy range. The fits were performed on H.E.S.S and Fermi-LAT eight spectrum points, as shown in Fig 3 . The fit parameters are reported in Table 3.1. The combined spectrum can be perfectly fit with a single power law, resulting in a reduced $\chi^{2}$ of 8.2 for 6 degrees of freedom (Fig. 3 left). The derived photon index, $\Gamma=2.13 \pm 0.05$, is consistent with the index obtained with the H.E.S.S. data alone. The fit model with a power law and an exponential cutoff (Fig. 3 right $), d N / d E=\Phi_{0} \times(E / 1 \mathrm{TeV})^{-\Gamma} \exp \left(-E / E_{\mathrm{c}}\right)$, is not preferred over a fit with a simple power law as derived from this table by means of a likelihood ratio test $\left(1.4 \sigma, \Delta \chi^{2}=1.8\right.$ with 1 degree of freedom). Considering the Akaike information criterion (AIC; Akaike 1974), an AIC value of 12.4 is obtained while fitting a power law with an exponential cutoff to the data, which is 12.2 when a power law is fit to the data. The relative likelihood defined as $\exp \left(\left(\mathrm{AIC}_{\min }-\mathrm{AIC}_{\max }\right) / 2\right)$ shows that the power-law model is sufficient to describe the data. The resulting cutoff energy is quite high, exhibiting large errors $\left(E_{c}=19_{-10}^{+60} \mathrm{TeV}\right)$. A cutoff energy lower than $8 \mathrm{TeV}$ can be excluded at a $95 \%$ con- fidence level (CL). The energy cutoff value is based on the statistical analysis and does not take into account the H.E.S.S. systematic error in the energy reconstruction of $\sim 15 \%$. A broken power-law fit to the joint Fermi/H.E.S.S. data yields values (Fig. 3 right) of the break energy between $8 \mathrm{GeV}$ and $140 \mathrm{GeV}$, with a best-fit value of $24 \mathrm{GeV}$, and it is as likely as a simple power law fit $\left(1.5 \sigma, \Delta \chi^{2}=4.0\right.$ with 2 degrees of freedom, and an AIC value of 12.2). These lower and upper bounds were established by fitting the data with a fixed value for the energy break until a $\Delta \chi^{2}=1$ was reached $(68 \% \mathrm{CL})$, leaving all other parameters free.

\subsection{Morphology in the VHE gamma-ray band}

The position of the VHE gamma-ray source was obtained from a two-dimensional fit of the H.E.S.S. so-called ON map consisting of gamma-ray-like events in the source region and a background map, using the gamma-ray events selected in the OFF region multiplied by a scaling factor map in order to reach the source exposure level. The fit was performed using the Sherpa package (Freeman et al. 2001), with a log-likelihood approach (Cash statistic; Cash 1979) and the Levenberg-Marquardt algorithm (Moré 1978). To obtain the source position, a Gaussian source was convolved with the H.E.S.S. analysis PSF (0.06) multiplied by the exposure map to take any asymmetry in the exposure across the analyzed sky region into account, and with the background amplitude fixed to 1 . As there is no evidence for an extension, a point source assumption was used by fitting the two-dimensional Gaussian profile with a width of $\sigma=0.005$. The fit results in a best-fit position of RA $5^{\mathrm{h}} 24^{\mathrm{m}} 47^{\mathrm{s}} \pm 6.9_{\text {stat }}^{\mathrm{s}}$ and Dec $-69^{\circ} 38^{\prime} 50^{\prime \prime} \pm 29_{\text {stat }}^{\prime \prime}$ at about $1^{\prime}$ from the X-ray source position. The uncertainty of the telescope pointing leads to a systematic error of $\sim 20^{\prime \prime}$ per axis. The systematic error arising from the asymmetrical exposure is negligible. The best-fit position and the confidence region are shown in Fig. 1 and are consistent with the center of N132D as seen in X-rays within $\sim 1.2 \sigma$.

Given the diameter of N132D in X-rays of $1.6^{\prime}$ and the presence of a nearby molecular cloud at a distance of $\sim 2$ arcminutes, it is likely that the gamma-ray source has a structure on the arcminute scale. With the reported data, a two-dimensional fit with a Gaussian profile centered on the best-fit position provides only a $99 \% \mathrm{CL}$ upper limit on the source extension in gamma rays corresponding to $\sigma_{2 \mathrm{D}, \text { Gauss }}=3.3^{\prime}(49 \mathrm{pc})$. Because the PSF is $3.6^{\prime}$, the localization of the emitting region is inside this radius.

\section{Modeling the multiwavelength spectrum}

The results were analyzed in the context of the multiwavelength studies of N132D. Radio observations at wavelengths $3 \mathrm{~cm}$ and $6 \mathrm{~cm}$ by Dickel \& Milne (1995) with the Australia Telescope Compact Array (ATCA), and nonthermal X-ray upper limits in the 0.2 to $4 \mathrm{keV}$ band (Hughes et al. 1998) and in the 2-10 $\mathrm{keV}$ band provided by Bamba et al. (2018) were added to the gamma-ray spectrum. This led to a multiwavelength spectrum spanning 15 orders of magnitude (see Fig. 4). Radio and nonthermal X-ray emission can be related to gamma-ray emission based on two different scenarios. The leptonic scenario assumes that a single population of CR electrons generates synchrotron emission in the radio to X-ray range and inverse Compton emission in gamma rays. Alternatively, in the hadronic scenario, $\mathrm{CR}$ electrons are only responsible for the synchrotron radio and $\mathrm{X}$ ray emissions, while CR hadrons produce gamma-ray emission through neutral pions that decay into two photons. In this case, a ratio of $n_{\mathrm{e}} / n_{\mathrm{p}}$ for CRs is one of the parameters, for which the 


\begin{tabular}{|c|c|c|c|c|}
\hline Data & $\chi^{2} /$ ndof & $\Gamma$ & $\begin{array}{c}\Phi_{0}(1 \mathrm{TeV}) \\
\times 10^{-14} \mathrm{TeV}^{-1} \mathrm{~cm}^{-2} \mathrm{~s}^{-1}\end{array}$ & $E_{c} / E_{b}$ \\
\hline H.E.S.S. PL & $3.9 / 2$ & $2.32 \pm 0.22$ & $13.1 \pm 4.3$ & - \\
\hline Fermi-LAT + H.E.S.S PL & $8.2 / 6$ & $2.13 \pm 0.05$ & $9.7 \pm 1.6$ & - \\
\hline Fermi-LAT + H.E.S.S ECPL & $6.4 / 5$ & $2.08 \pm 0.06$ & $12.3 \pm 2.9$ & $E_{c}=19_{-10}^{+60} \mathrm{TeV}$ \\
\hline Fermi-LAT + H.E.S.S BPL & $4.2 / 4$ & $1.47 \pm 0.43 \quad 2.31 \pm 0.14$ & $12.8 \pm 2.9$ & $E_{b}=24_{-16}^{+110} \mathrm{GeV}$ \\
\hline
\end{tabular}

Table 1. Spectral fit parameters for the H.E.S.S. and Fermi-LAT plus H.E.S.S. data sets. PL stands for "power law", ECPL for "power law with an exponential cutoff", and "BPL" for "broken power law" (see text for details).
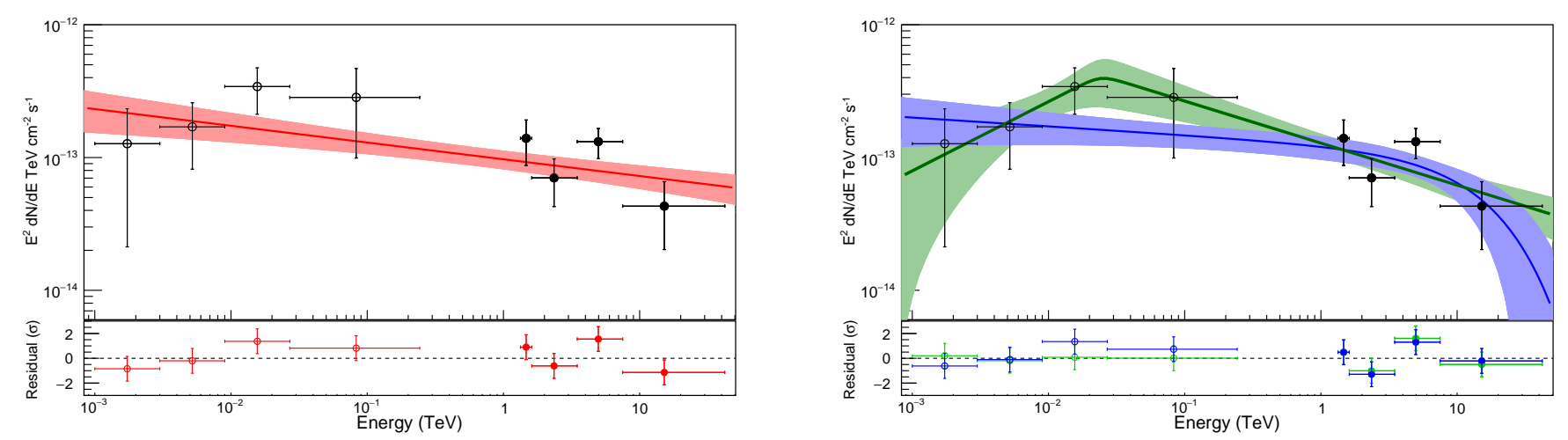

Fig. 3. Left: Fermi-LAT (open circles) and H.E.S.S (dots) combined spectrum fit with a power law. Right: Fermi-LAT and H.E.S.S combined spectrum fit with a power law with an exponential cutoff (blue) and a broken power law (green). For both figures, the best-fit functions are shown with $68 \%$ error bars in the shaded region, as well as residuals compared to the fit. The flux point error bars represent the statistical uncertainties. The fit parameters are reported in Table 3.1

canonical value is $1 / 100$, based on the ratio of electron-to-proton CRs measured near the Earth (see, e.g., Longair 2011; Katz \& Waxman 2008). However, the $n_{\mathrm{e}} / n_{\mathrm{p}}$ ratio at Earth may not reflect the ratio in SNRs, and there may be variations in SNRs. Here these radiation processes were modeled using theoretical frameworks developed by Aharonian et al. (2010) for the synchrotron radiation, by Khangulyan et al. (2014) for the inverse Compton mechanism, by|Baring et al. (1999) for bremsstrahlung emission, and by Kafexhiu et al. (2014) for the pion decay. To model the multiwavelength spectrum of N132D for the two scenarios, the Naima package was employed (Zabalza 2015). We used a single-zone model. In reality, emission may arise from a number of regions, such as the shocked ejecta, the main shell of the shocked ambient medium, or even, as we discuss below, from cosmic rays interacting with nearby molecular clouds.

\subsection{Purely leptonic model}

The multiwavelength data can be reasonably well modeled with a purely leptonic model assuming synchrotron emission with a magnetic field of $20 \mu \mathrm{G}$ and inverse Compton emission with two background radiation components, corresponding to the cosmic microwave background (CMB) radiation with an energy density of $0.26 \mathrm{eV} \mathrm{cm}^{-3}$ and an IR component intrinsic to the remnant itself with a temperature of $145 \mathrm{~K}$ and an energy density of 1 $\mathrm{eV} \mathrm{cm}^{-3}$ (see Abramowski et al. 2015b). The latter component is due to dust emission from and around the SNR. The model assumes an electron distribution following a power law with an exponential cutoff. Fig. 4 shows the model, and the corresponding parameters are reported in Table 4.1 For this model, the required total electron energy is $\mathrm{W}_{e}(>1 \mathrm{GeV})=4.50 \times 10^{49} \mathrm{erg}$, which is significantly higher than observed in other well-studied SNRs (as discussed in section 5.2). Even if an initial released en- ergy higher than the canonical value is considered, for instance, $\sim 5 \times 10^{51}$ erg (see, e.g., Bamba et al. 2018), then $10 \%$ of this energy can be transmitted to CR protons, which would imply a maximum electron energy of $\mathrm{W}_{e} \simeq 5 \times 10^{48} \mathrm{erg}$ if $n_{e} / n_{p}=0.01$ is assumed.

Diffusive shock acceleration should lead to an initial particle distribution that is a power law in momentum (e.g., Malkov \& Drury 2001). This means that the energy distribution breaks around the rest-mass energy of the particles. Hence, diffusive shock acceleration produces an electron spectrum that is expected to continue as an unbroken power law to energies well below $1 \mathrm{GeV}$ (e.g. Asvarov et al. 1990; Vink 2008), unlike the hadronic CR distribution. For example, if a lower limit at 10 $\mathrm{MeV}$ is considered, then $\mathrm{W}_{e}(>10 \mathrm{MeV})=1.3 \times 10^{50} \mathrm{erg}$, which suggests that an implausibly high fraction of $>2 \%$ of the explosion energy would be contained in relativistic electrons.

The value of $20 \mu \mathrm{G}$ for the magnetic field strength required by the leptonic model is similar to what was inferred by Abramowski et al. (2015b) and Bamba et al. (2018). Although some 1000-3000 yr old SNRs do have similar magnetic fields (Bamba et al. 2005, Helder et al. 2012, Zeng et al. 2019), such a magnetic field strength is surprisingly low for a radio source as luminous as N132D ( 238 times more luminous at $1 \mathrm{GHz}$ than the $\sim 1835$ yr old SNR RCW 86 , which is a VHE $\gamma$-ray source), and this would imply that the magnetic field energy density is far out of equipartition with relativistic particle energy density. The equipartition magnetic field strength can be derived from the minimum energy principle, which minimizes the total energy of the nonthermal particles plus the magnetic field energy for a given radio luminosity. The magnetic field strength thus derived, $\mathrm{B}_{\text {min }}$, is close to the equipartition value (Burbidge 1956). It leads to the following relation for $\mathrm{B}_{\min }$ (for a review, see Longair 2011, Vink 2020): $B_{\min }(\mathrm{G})=9.3 \times 10^{3}\left(\frac{\eta L_{v}}{V}\right)^{2 / 7} v^{1 / 7}$, with $L_{v}$, the radio 
luminosity in erg s $\mathrm{s}^{-1} \mathrm{~Hz}^{-1}$ at a frequency $v$ in $\mathrm{Hz}, V$ corresponds to $25 \%$ of the volume in $\mathrm{cm}^{3}$ of a sphere with the diameter of $\mathrm{N} 132 \mathrm{D}(28 \mathrm{pc})$, and $\eta$ is a parameter taking into account the ratio of the energy present in CR nuclei versus electrons $(1<\eta<100)$. Considering the radio luminosities reported by Dickel \& Milne (1995) $\left(L_{v}=4.3 \times 10^{24} \mathrm{erg} \mathrm{s}^{-1} \mathrm{~Hz}^{-1}\right.$ at $\left.5 \mathrm{GHz}\right)$ and the volume corresponding to a diameter of $28 \mathrm{pc}, B_{\text {min }} \approx 35 \mu \mathrm{G}$ is obtained for $\eta=1$ (the relativistic electron density equals that of CR nuclei), and $134 \mu \mathrm{G}$ for $\eta=100$.

Fig. A.1 shows the purely leptonic model with bremsstrahlung in addition to the synchrotron and inverse Compton emission for the electron distribution following a power law with an exponential cutoff as described above. For the bremsstrahlung component to be important, the density of the proton targets has to be as high as $n_{\mathrm{p}}=3 \mathrm{~cm}^{-3}$. At this high density, the pion decay process will play a considerable role, which violates the assumption of a pure leptonic model. In a purely leptonic scenario, bremsstrahlung emission cannot explain the data if the proton density remains low. In the case of a mixed lepto-hadronic model as described in the next section, a hadronic component would require a lower energy for the protons and electrons, leading to a higher magnetic field value. Then bremsstrahlung emission is marginal and not needed to explain the data. It can be concluded here that a purely leptonic model is unrealistic for the HE-VHE gamma-ray emission considering the extreme total energy in electrons required to explain the measured spectra, and a dominant component of hadronic origin is required.

\subsection{Hadronic model}

The HE-VHE gamma-ray spectrum can be well fit with a pion decay model, assuming a proton distribution following a power law with an exponential cutoff. The model requires an ambient proton density of $n_{\mathrm{p}}=10 \mathrm{~cm}^{-3}$ (Hughes et al. 1998), a total CR proton energy $\mathrm{W}_{p}(>1 \mathrm{GeV})=4 \times 10^{50} \mathrm{erg}$, a power-law spectral index $\Gamma=2.1$, and a cutoff energy of $E_{c}=120 \mathrm{TeV}$ (Table 4.1). The cutoff energy value is remarkably high: a lower limit is set at $E_{c}=45 \mathrm{TeV}$ with $95 \% \mathrm{CL}$, which is much higher than the cutoff in the proton spectrum for Cas A, which is estimated to be at $\sim 10 \mathrm{TeV}$ by Ahnen et al. (2017). The total energy in CR protons, $W_{p}(>1 \mathrm{GeV})=4 \times 10^{50} \mathrm{erg}$, is high but not unrealistic because the energy released in the explosion is thought to exceed $10^{51}$ erg (e.g., Dickel \& Milne 1995), $10 \%$ of which can be transferred to hadronic CRs. The pion decay fit can be achieved with a lower CR proton energy and a higher ambient proton density, for example, $W_{p}(>1 \mathrm{GeV})=1 \times 10^{50} \mathrm{erg}$ and $n_{p}=40 \mathrm{~cm}^{-3}$, but higher proton densities would necessitate additional target material, other than the SNR itself, for the hadronic interaction to take place, for example, a nearby molecular cloud.

Radio and X-ray data were fit with a synchrotron emission model assuming that the electron energy distribution follows a power law with an exponential cutoff, the normalization being constrained by a total electron energy $W_{e}(>1 \mathrm{GeV})=4 \times 10^{48} \mathrm{erg}$, assuming $1 / 100$ of the proton energy. The model requires a high magnetic field strength in order to make the leptonic gamma-ray emission subdominant, set here to $B=100 \mu \mathrm{G}$, with a cutoff in the electron distribution at $\mathrm{E}_{c}(100 \mu \mathrm{G}) \leqslant 3.5 \mathrm{TeV}$, in order to fit the radio data points and ensure that the X-ray synchrotron component does not exceed the X-ray upper limits. For the spectrum presented in Fig. 4. $\mathrm{E}_{c}=2.5 \mathrm{TeV}$ was chosen (see parameters in
Table 4.1). In this model, a cutoff energy in the proton distribution is significantly higher than that in the electron distribution ${ }^{4}$

Our model is compatible with similar models in the literature, such as those proposed for Cas A by Abeysekara et al. (2020), and to some extent, the model for RX J1713.7-3946 (see Abdalla et al.2018d), both of which normalize the electron distribution to the radio and X-ray synchrotron emission. The chosen magnetic field strength is close to the equipartition value of $135 \mu \mathrm{G}$. The $W_{e} / W_{p}$ ratio depends on the electron-to-proton $\left(n_{e} / n_{p}\right)$ ratio, but also on the respective volumes for integrating proton and electron densities, leading to total energy values. CR electrons can fiducially be considered to emit synchrotron radiation within the SNR volume.

If gamma-ray emission from both $\mathrm{CR}$ electrons and protons originates within the $\mathrm{SNR}$, then the assumption of $\mathrm{W}_{e} / \mathrm{W}_{p}=$ $n_{e} / n_{p}=1 / 100$ is reasonable. However, if gamma-ray emission is mainly produced by escaped $\mathrm{CR}$ protons interacting with a molecular cloud, then the $\mathrm{W}_{e} / \mathrm{W}_{p}$ ratio can have a different value.

\section{Discussion}

The analysis of 252 hours of H.E.S.S. observations of N132D confirms the previously published result that N132D is an exceptionally luminous SNR in the VHE band. The well-determined distance to the LMC makes our luminosity estimation robust; it is therefore worthwhile to compare the gamma-ray SED of N132D to the SEDs of the most luminous SNRs in gamma rays. We found that only one Galactic SNR, G338.3-0.0, is firmly established to show a VHE luminosity that is comparable to that of N132D (see Appendix B for details). G338.3-0.0 is a composite SNR identified with the VHE source HESS J1640-465, but it is not clear whether the VHE emission comes predominantly from the central PWN or the SNR shell (Abdalla et al.2018a).

Another important and new result of the analysis presented here is that no clear cutoff has been found in the gamma-ray spectrum, which extends up to $15 \mathrm{TeV}$. The last bin of the spectrum presented in Fig. 3 corresponds to gamma rays with energies of about $10 \mathrm{TeV}$ or beyond, which is remarkable for such a distant SNR. The spectra of only a few SNR shells reach such a high energy, and they are younger and more nearby sources than N132D. Assuming a hadronic origin for the gamma-ray emission from N132D, this implies that protons are present in N132D with energies of at least one hundred $\mathrm{TeV}$.

The extremely high VHE luminosity and the extension of the high-energy power-law tail to $15 \mathrm{TeV}$ are two observational properties that need to be examined in the context of the physics of SNRs and their environment. For this purpose, the VHE gamma-ray properties of N132D were compared with those of SNRs with similar physical properties, such as age, shock speed, and circumstellar structure (stellar wind bubbles and vicinity of molecular clouds).

\subsection{Comparison of properties among SNRs}

Supernova remnants are thought to be efficient accelerators during time intervals when the shock speed is high and they transit from the ejecta-dominated phase to the Sedov phase. SNR RX J1713.7-3946 is one of the brightest VHE-emitting SNR and is often considered as a prototypical CR accelerator. It is younger

\footnotetext{
${ }^{4}$ In reality, the electron spectrum may even have a break well below $2.5 \mathrm{TeV}$ as a cooling break is expected at $\sim 0.5 \mathrm{TeV}$ for $t=2500 \mathrm{yr}$ and $B=100 \mu \mathrm{G}$ (see Sect. 13.3 Vink 2020).
} 


\begin{tabular}{cccccccc}
\hline MODEL & $\begin{array}{c}\mathrm{W}_{e}(>1 \mathrm{GeV}) \\
\mathrm{erg}\end{array}$ & $\Gamma$ & $\begin{array}{c}E_{c_{\text {elec }}} \\
\mathrm{TeV}\end{array}$ & $\begin{array}{c}\mathrm{B} \\
\mu \mathrm{G}\end{array}$ & $\begin{array}{c}\mathrm{W}_{p}(>1 \mathrm{GeV}) \\
\mathrm{erg}\end{array}$ & $\begin{array}{c}\mathrm{n}_{p} \\
\mathrm{~cm}^{-3}\end{array}$ & $\begin{array}{c}E_{c_{\text {prot }}} \\
\mathrm{TeV}\end{array}$ \\
\hline Leptonic & $\begin{array}{c}4.5 \times 10^{49} \\
4 \times 10^{48}\end{array}$ & 2.2 & 8 & 20 & - & - & - \\
Hadronic & 2.1 & 2.5 & 100 & $4 \times 10^{50}$ & 10 & 120 \\
\hline
\end{tabular}

Table 2. Parameters for the proposed models of the N132D multiwavelength spectrum. For details, see text.
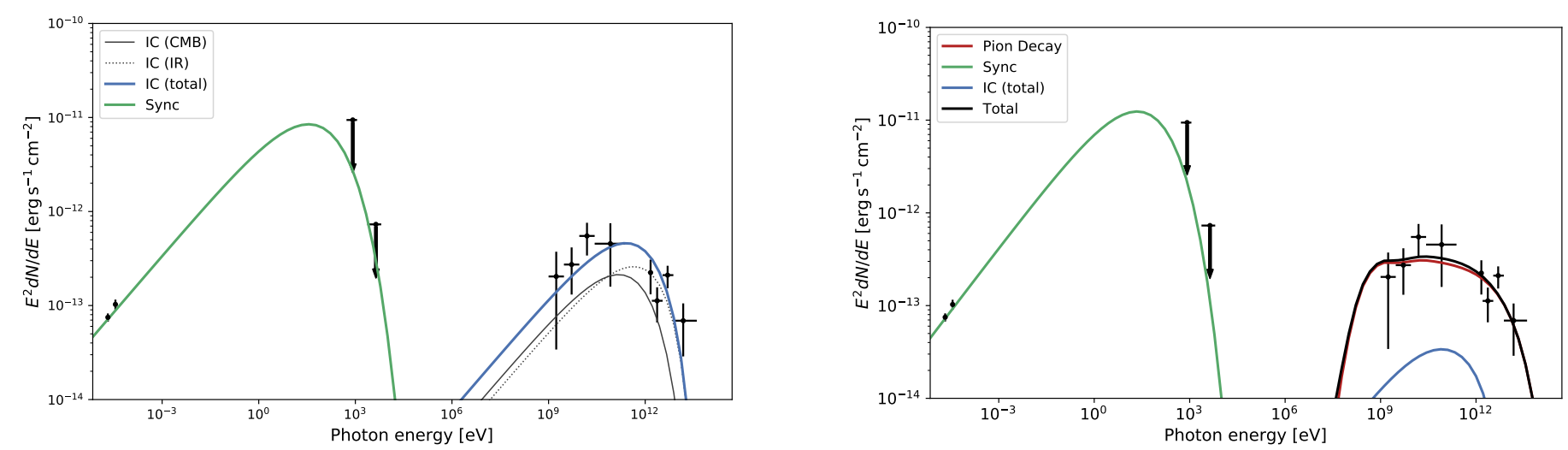

Fig. 4. Left: Leptonic model considering an electron distribution following a power law with an exponential cutoff. The parameters are described in Table 4.1 and the IR photon density is $1 \mathrm{eV} \mathrm{cm}^{-3}$. Right: Hadronic model considering a proton distribution following a power law with an exponential cutoff. The parameters are described in Table 4.1

(1600-2100 yr, e.g., Tsuji \& Uchiyama 2016) than N132D and it is a nearby source (at $1 \mathrm{kpc}$ ). It has a high shock speed of $\sim 3500$ $\mathrm{km} \mathrm{s}^{-1}$, therefore the conditions are fulfilled for rapid diffusive shock acceleration. Due to its gamma-ray brightness, its spectrum is measured well beyond $10 \mathrm{TeV}$, and it exhibits a cutoff at $E_{\mathrm{c}}=12.9 \pm 1.1 \mathrm{TeV}$ (Abdalla et al.2018d). For this source, the leptonic scenario is as plausible as the hadronic one. For a hadronic scenario, the cutoff in the parent proton distribution is estimated at $E_{c}=88 \mathrm{TeV}$, which is similar to our results. With an age of about $2500 \mathrm{yr}, \mathrm{N} 132 \mathrm{D}$ is about an order of magnitude more luminous in the VHE band than SNR RX J1713.73946. Thus, even if the age of SNRs is one of the factors making N132D a source with a high CR energy content, other factors should also play a role in making this source so luminous. The X-ray emission from RX J1713.7-3946 is dominated by synchrotron radiation from $10-100 \mathrm{TeV}$ electrons, whereas there is no evidence for X-ray synchrotron emission from N132D, whose $\mathrm{X}$-ray emission is dominated by thermal radiation. Figure 5 shows the differential luminosities for five SNRs demonstrating that N132D is one of the two most luminous SNRs, together with HESS J1640-465. In all these VHE sources, only the distant but luminous N132D and the nearby RX J1713.7-3946 exhibit flux points above $10 \mathrm{TeV}$.

The gamma-ray emission properties of SNRs have recently been compared by Zeng et al. (2019) and Suzuki et al. (2020) using large SNR samples. A comparison of these results to the properties of N132D presented here confirms the exceptional nature of N132D. In particular, the inferred gamma-ray cutoff energy of $E_{c} \gtrsim 8 \mathrm{TeV}$ is higher than those of the SNRs in Fig. 5b in Suzuki et al. (2020), who used a spectral model comparable to the one used by us. Similar conclusion can be drawn from Fig. 3 of Zeng et al. (2019), however, care should be taken wherever a lower limit to the cutoff energy is provided for spectra with a broken power-law shape, as this is an additional model complexity.

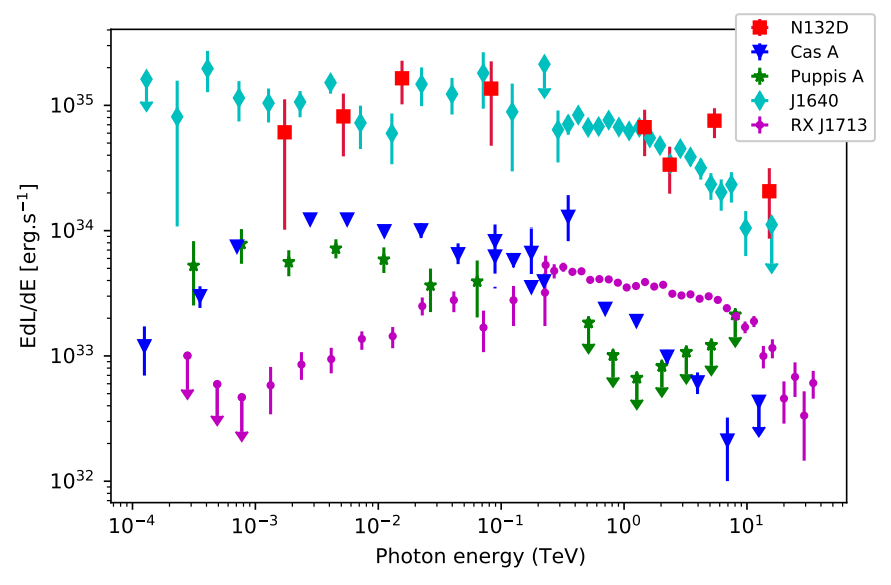

Fig. 5. Comparison of differential luminosities, EdL/dE, for VHE bright SNRs. Luminosities are computed using the differential flux data points and estimated distances in the following references: N132D, this work, $\mathrm{d}=50 \mathrm{kpc}$; Cas A, Ahnen et al. (2017), $d=3.4 \mathrm{kpc}$; Puppis A , Abramowski et al. (2015a), $d=2.2 \mathrm{kpc}$; HESS J1640-465, LemoineGoumard et al. (2014), $d=10 \mathrm{kpc}$; and RX J1713.7-3946, Abdalla et al. (2018d), $d=1 \mathrm{kpc}$. The different shapes of the individual SEDs probably reflect the different emission mechanisms (leptonic or hadronic) and evolutionary stages. See the original references for detailed spectral models.

N132D belongs to the small class of oxygen-rich SNRs (see Vogt \& Dopita 2011), together with Cas A and Puppis A. These SNRs are characterized by elevated abundances of oxygen, neon, and other heavy elements, indicating that this material has its origin within the helium-burned layers of massive progenitor stars. For the progenitor of N132D, different values for its mass have been suggested, for instance, $>35 M_{\odot}$ (France et al. 2009) and $15 \pm 5 M_{\odot}$ (Sharda et al. 2020), while the progenitors of Cas A and Puppis A have masses in the range of $15-25 M_{\odot}$ (Young et al. 
2006; Hwang et al.2008). The comparison of N132D with Cas A shows that the two remnants have similar properties in the radio and X-ray bands, and bright oxygen emission. The distance of Cas A is measured to be $3.4 \mathrm{kpc}$ (Reed et al. 1995), and its age is $\simeq 340$ years (Thorstensen et al. 2001). N132D is therefore much older than Cas A. However, Fig. 5 clearly shows that N132D is much more luminous than its younger sibling. As already mentioned, the Cas A spectrum exhibits a cutoff at $3.5 \mathrm{TeV}$ that is absent in the N132D spectrum. Puppis A is an older oxygen-rich SNR with an age estimated to be $4450 \pm 750$ years and a distance of $2.2 \pm 0.3 \mathrm{kpc}$. It is very similar to N132D in terms of chemical composition and age, but it is 30 times closer. Puppis A has not been detected in VHE gamma rays (Abramowski et al. 2015a), and its $\mathrm{GeV}$ emission is thought to be due to the interaction with a nearby molecular cloud. A recent study concluded that there is a spectral break at $\mathrm{GeV}$ energies, which could explain the absence of VHE emission (Xin et al. 2017). In this regard, Puppis A could be similar to the mature SNRs W44 or IC443, whereas, as demonstrated by Bamba et al. (2018), N132D could be in the transition from being young to a intermediate-age SNR. These facts show that there is significant variation in the observational gamma-ray properties among the members of the oxygen-rich SNR class.

\subsection{Interstellar environment}

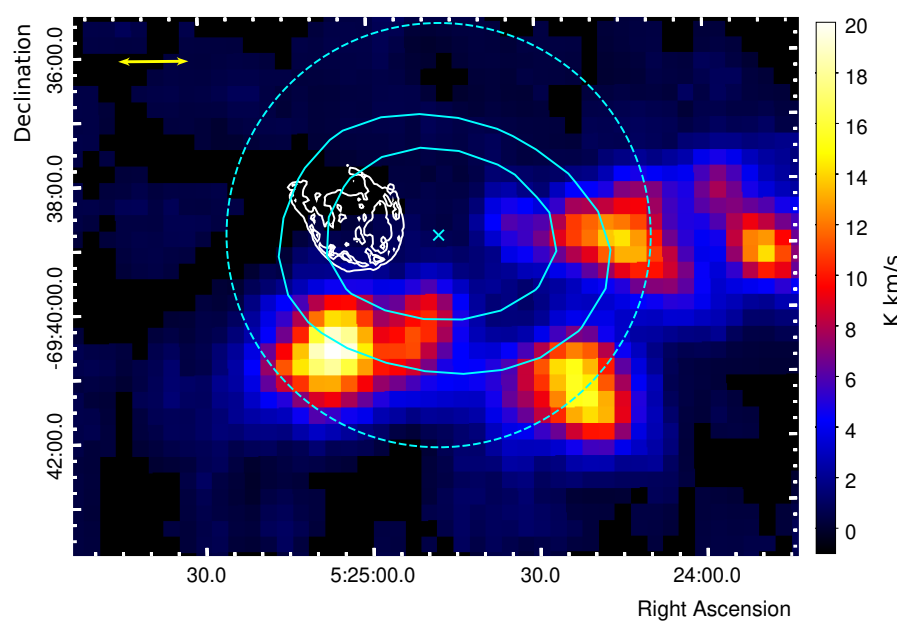

Fig. 6. Molecular clouds in the vicinity of N132D. The image shows the $\mathrm{CO}$ emission (color scale in $\mathrm{K} \mathrm{km} / \mathrm{s}$ ) from the MAGMA survey ${ }^{5}$ (Wong et al. 2011). White contours denote Chandra X-ray emission from N132D (Borkowski et al. 2007). The best-fit position of the gamma-ray emission is denoted by the cross, and the elliptical contours are the confidence regions of the position at $2 \sigma$ and $3 \sigma$. The dashed circle illustrates the $99 \%$ CL UL on the extension established at 3.3'. The scale bar indicates $1^{\prime}$

For hadronic emission, the VHE luminosity is determined by the total energy in CRs above $\sim 10 \mathrm{TeV}$ and the local gas density. The high TeV gamma-ray luminosity of N132D may therefore reflect a combination of a high energy in CRs, perhaps caused by a relatively high explosion energy for this SNR, and a high gas density. Two possible locations of a dense gas are discussed below, including the shell of a wind-blown cavity and a massive molecular cloud near N132D. Our multiwavelength modeling indeed suggests that the gamma-ray emission most likely has a hadronic origin. In the case of a leptonic scenario, the required total energy in the electrons is high com- pared with other observed SNRs (see also Bamba et al. 2018). For example, a leptonic model for the gamma-ray emission from SNRs with a low GeV-to-TeV flux ratio, RX J1713.7-3946 and RX J0852.0-4622 (e.g., see Fig. 3 in Dermer \& Powale 2013), implies a total electron energy, $W_{\mathrm{e}}(\mathrm{E}>1 \mathrm{GeV})$, of $3.1 \times 10^{47}$ erg (Abdalla et al. 2018d) and $2.24 \times 10^{48}$ erg (Abdalla et al. 2018e), respectively. For HESS J1640-465, a similar modeling leads to $W_{\mathrm{e}}=10^{49} \mathrm{erg}$ (Abramowski et al. 2014). To a lesser extent, the lack of nonthermal X-rays from N132D is also in favor of a hadronic origin, although nonthermal X-ray emission may be hidden below the bright thermal X-ray continuum. An X-ray synchrotron luminosity similar to that of RX J1713.7-3946 cannot be excluded. Moreover, the radio luminosity of N132D suggests a high magnetic field strength, compatible with the $100 \mu \mathrm{G}$ strength assumed in the hadronic model presented in section 4.2

For the preferred hadronic scenario for the gamma-ray emission of N132D, a high gas density is required, and the question arises where most of these gamma rays are coming from: from CRs within the densest portions of the SNR shell, or from CRs that have escaped the shell and are interacting with the massive molecular cloud near N132D (Banas et al. 1997; Sano et al. 2020). While the GeV luminosities of SNRs interacting with molecular clouds are higher than those of SNRs without nearby molecular clouds, this tendency does not hold for VHE gamma rays (e.g., Fernandez et al. 2013). The VHE luminosity enhancement for N132D and its hard gamma-ray spectrum at VHE energies can be interpreted under the assumptions that (1) the shock accelerates protons until the highest possible energy is reached, and at this point, the shock can no longer confine the protons, and (2) the most energetic protons escape then into the interstellar medium (ISM). The gamma-ray emission created by the interactions of these escaped protons with a gas cloud will be strong and hard (Aharonian \& Atoyan 1996, Gabici \& Aharonian 2007; Moskalenko et al. 2008). The hadronic scenario requires a high density of the target material. These high densities can be found in molecular clouds. SNRs such as W28 or W44 show gamma-ray emission originating in nearby molecular clouds (see, e.g., Aharonian et al. 2008, Cardillo et al. 2014, Makino et al. 2019), indicating that protons accelerated in the SNR bombard the molecular cloud. N132D is interacting with a molecular cloud as well, which has recently been shown by Sano et al. (2020). Furthermore, the X-ray emission is brightest in the southwestern part, where the SNR is interacting with dense matter.

The molecular clouds near N132D as traced by the CO emission are shown in Fig. 6. The velocity of the clouds, $+264 \mathrm{~km}$ $\mathrm{s}^{-1}$, agrees with that of $\mathrm{N} 132 \mathrm{D},+268 \mathrm{~km} \mathrm{~s}^{-1}$ (Banas et al. 1997). The best-fit position of the gamma-ray emission (elliptical contours in Fig. 6) is consistent with the position of the SNR itself and with parts of the molecular cloud. Therefore these two regions are potential sites of gamma-ray emission. If the VHE gamma-ray emission comes from these molecular clouds, a diffusion coefficient of $D \simeq 2 \times 10^{29}(t / 2500 \mathrm{yr})^{-1} \mathrm{~cm}^{2} \mathrm{~s}^{-1}$ for multi-TeV CRs is required. To determine the spatial origin of the gamma-ray emission, a higher sensitivity and a finer angular resolution are needed. Nevertheless, the spectrum with an unbroken power law from $\mathrm{GeV}$ to $\mathrm{TeV}$ energies could be taken as evidence for a single population of emitting particles. This would be difficult to reconcile with illumination of MCs by CRs originating from the shell. In that case, the low-energy gamma rays would likely come from the shell because only the highest-energy CRs would penetrate the MC and cause gamma-ray radiation at VHE. In addition to the molecular clouds shown in Fig. 6, Sano et al. (2020) reported smaller clouds that interact with the SNR, as 
indicated by the ratio of CO $J=3-2$ versus $J=1-0$ line emission. These cloudlets are situated in the south and near the center of the SNR. They might also be locations of enhanced hadronic emission, but then directly associated with the SNR itself.

A hadronic scenario involving a wind-blown cavity is also possible. As suggested by Chen et al. (2003), in case of a very massive progenitor, a low-density bubble is formed (see also Weaver et al. 1977). When the SN occurs, the shock propagates with a high velocity $\left(v \sim 5000-10000 \mathrm{~km} \mathrm{~s}^{-1}\right)$ into the bubble. When the shock wave reaches the high-density shell of swept-up material, it heats the dense shell, and the SNR appears most luminous, but the shock velocity rapidly decreases. A wind-blown bubble model was considered for G338.3-0.0 in order to explain the high VHE luminosity of HESS J1640-465 (Abramowski et al.2014). Under the assumptions that the SNR shock freely expands up to the radius of the wind-blown bubble, and with average shock speeds between $5000 \mathrm{~km} \mathrm{~s}^{-1}$ and 10000 $\mathrm{km} \mathrm{s}^{-1}$, the authors proposed that the age of SNR G338.3-0.0 could be $1000-2000 \mathrm{yr}$ in this case, and at least younger than 5000-8000 yr (Slane et al. 2010).

The wind-blown bubble model also fits the properties of $\mathrm{N} 132 \mathrm{D}$, given the inconsistency between its kinematic age (2500 $\mathrm{yr})$ and its Sedov dynamical age $(6000 \mathrm{yr})$, indicating that the supernova explosion occurred within a cavity in the interstellar medium (Hughes 1987). In addition to the VHE luminosity enhancement, it is conceivable that this model can also explain the presence of sub-PeV protons to some extent because an SNR expanding into a preexisting stellar wind can accelerate protons to a higher energy than SNRs expanding in a uniform circumstellar medium (Völk \& Biermann 1988). Because the gammaray spectrum of G338.3-0.0/HESS J1640-465 has an exponential cutoff at $6 \mathrm{TeV}$, it is unclear so far whether the luminosity enhancement and the increase in a maximum CR energy are expected to be observed simultaneously.

Two other SNRs in the LMC, N63A and N49B, have also been proposed to have evolved in wind-blown cavities (Hughes et al. 1998). Because N63A and N49B were detected with FermiLAT (Campana et al. 2018) and have massive progenitors, it is possible that the luminosity enhancement takes place in these SNRs as well, as suggested, for example, in Sano et al. (2017). This hypothesis can be tested by observations of N63A and $\mathrm{N} 49 \mathrm{~B}$, which are located in the northern part of the LMC disk about $4^{\circ}$ away from N132D.

In general, the LMC is well suited for studies of SNRs (see Ginzburg 1972) because its projection is almost face-on (inclination of $35^{\circ}$ ) and the distance to the LMC SNRs is more certain than for some Galactic SNRs, allowing us to accurately derive the VHE luminosity. Galactic PeVatron candidates with hard $(\Gamma \sim 2)$ and featureless spectra similar to those derived for N132D in this work can be listed among unresolved H.E.S.S. sources in the Galactic plane (e.g., Spengler 2020). For example, SNR G318.2+0.1, one of the two most luminous Galactic SNRs at VHE, was proposed to be associated with HESS J1457-593 in 2010 (Hofverberg et al. 2010a) and is listed among PeVatron candidates. However, the association of Galactic VHE sources is often complicated by the presence of several possible source candidates for a given region within the Galactic plane.

Because of the lack of evidence for an energy cutoff in the N132D spectrum, N132D remains a PeVatron candidate. Cristofari et al. (2020) suggested that only the remnants of very powerful rare supernova explosions such as the one that produced the N132D remnant can accelerate CR particles to PeV energies. Moreover, the LMC has a significant angular size, allowing a detailed study of individual SNRs resolved in the VHE band.
Identifying the origin of CR hadrons is a long-standing problem (Ginzburg 1972), and the establishment of discrete accelerators of CR protons in the LMC, such as N132D, shows that SNRs are viable sources of CRs in the Milky Way and the LMC.

The possible role of molecular cloud interaction on the gamma-ray emission is worth investigating with the next generation of IACTs, in particular, with the Cherenkov Telescope Array (CTA; Acharya et al. 2019), which will have a sensitivity that is an order of magnitude better than H.E.S.S. and its angular resolution will be higher. Future N132D observations with CTA might therefore establish whether there is a shift between the SNR center and the origin of gamma-ray emission, and moreover, they might help to determine the energy of the spectral cutoff or even establish this enigmatic SNR as a CR PeVatron.

\section{Conclusion}

The LMC SNR N132D is detected with a statistical significance of $5.7 \sigma$ above $1.3 \mathrm{TeV}$ on the basis of H.E.S.S. observations with an exposure time of 252 hours, which is 104 hours longer than the exposure used in the previous publication Abramowski et al. 2015b). The inclusion of new observations results in an unambiguous detection and allowed us to perform a more detailed spectral analysis.

The Fermi-LAT and H.E.S.S. gamma-ray spectrum extends up to $15 \mathrm{TeV}$ and is well described with a power-law index of $2.13 \pm 0.05$. No cutoff in energy is needed to explain the spectrum, as a power law with an exponential cutoff fit is statistically as valid as a simple power-law model. A 95\% CL lower limit on an exponential cutoff is derived at $8 \mathrm{TeV}$. This lower limit exceeds the cutoff value of $3.5 \mathrm{TeV}$ established for the spectrum of 340-year-old Cas A by Ahnen et al. (2017), although N132D is $\sim 2500$ years old. N132D is the only extragalactic SNR detected in gamma rays so far, and its luminosity is compatible with that of the most luminous Galactic SNR G338.3-0.0. The absence of a clear spectral break and/or cutoff energy and its high gammaray luminosity make N132D a very special object. It is one of the oldest and most distant SNRs emitting at VHE.

A purely leptonic model fails to satisfactorily explain the multiwavelength spectrum of N132D. The main argument is that the required total energy of electrons is too high compared to the fraction of explosion energy that is expected to be transferred to leptonic CRs. An additional but less compelling argument is that the magnetic field strength in the case of a purely leptonic scenario is required to be surprisingly low, $20 \mu \mathrm{G}$, which is at odds with the radio brightness of this SNR. Finally, the cutoff energy required for the electron distribution is quite low $(8 \mathrm{TeV})$ compared to the absence of a cutoff observed in the gamma-ray spectrum. Our conclusion is that gamma-ray emission from N132D is most likely hadronic in origin.

The hadronic origin of the VHE gamma-ray emission raises the question whether its high luminosity is due to a very efficient acceleration at the SNR shock front, perhaps related to the evolution of the shock inside a wind-blown cavity, or to an interaction of the SNR with the molecular cloud, which could enhance its gamma-ray luminosity. A massive molecular cloud is present in the southwest of the remnant, where enhanced emission in radio, optical, and X-ray bands is observed. These two scenarios are possible given the uncertainties in VHE source position. Even if some of the emission is caused by $\mathrm{CR}$ interaction with the molecular cloud, the hard power-law spectrum, together with the lack of evidence for a cutoff energy, makes the gamma-ray SNR very distinct from mature SNRs that are known to interact with molecular clouds, such as IC 443, W44, and W28. 


\section{Acknowledgements}

The support of the Namibian authorities and of the University of Namibia in facilitating the construction and operation of H.E.S.S. is gratefully acknowledged, as is the support by the German Ministry for Education and Research (BMBF), the Max Planck Society, the German Research Foundation (DFG), the Helmholtz Association, the Alexander von Humboldt Foundation, the French Ministry of Higher Education, Research and Innovation, the Centre National de la Recherche Scientifique (CNRS/IN2P3 and CNRS/INSU), the Commissariat à l'énergie atomique et aux énergies alternatives (CEA), the U.K. Science and Technology Facilities Council (STFC), the Knut and Alice Wallenberg Foundation, the National Science Centre, Poland grant no.2016/22/M/ST9/00382, the South African Department of Science and Technology and National Research Foundation, the University of Namibia, the National Commission on Research, Science \& Technology of Namibia (NCRST), the Austrian Federal Ministry of Education, Science and Research and the Austrian Science Fund (FWF), the Australian Research Council (ARC), the Japan Society for the Promotion of Science and by the University of Amsterdam. We appreciate the excellent work of the technical support staff in Berlin, Zeuthen, Heidelberg, Palaiseau, Paris, Saclay, Tübingen, and in Namibia in the construction and operation of the equipment. This work benefitted from services provided by the H.E.S.S. Virtual Organisation, supported by the national resource providers of the EGI Federation. J. Vink and D. Prokhorov are partially supported by funding from the European Union's Horizon 2020 research and innovation programme under grant agreement No 101004131 and by the Netherlands Research School for Astronomy (NOVA). 


\section{Appendix A: Supplementary material for the multiwavelength study}

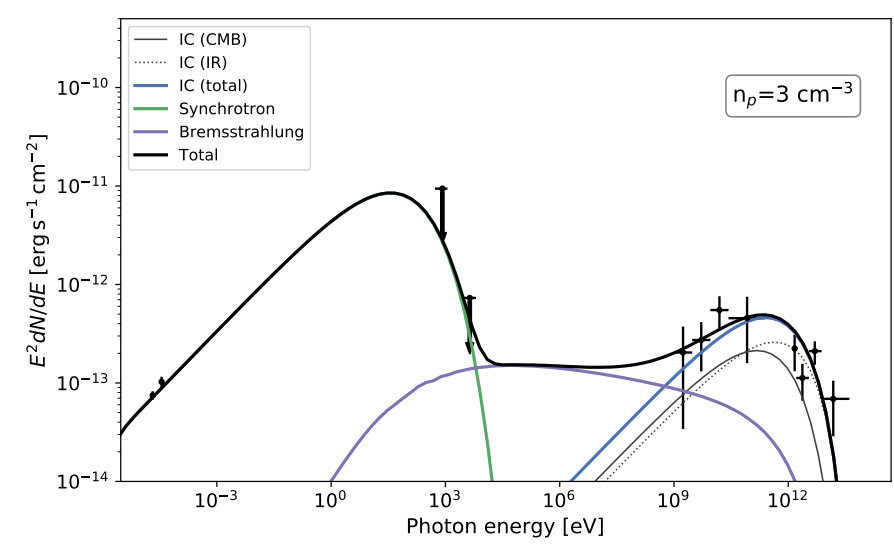

Fig. A.1. Leptonic model including bremsstrahlung emission. The model considered an electron distribution following a power law with an exponential cutoff. The parameters are described in Table 4.1 .

\section{Appendix B: Galactic SNRs}

The list of luminous (above $20 \%$ of the VHE luminosity of N132D) Galactic SNRs available in the literature includes CTB 37A, SNR G318.2+0.1, CTB 37B, SNR G359.1-0.5, SNR G106.3+2.7, SNR G8.7-0.1, and SNR G338.3-0.0, which are sorted here with increasing VHE luminosity. While this list consists of seven SNRs, there are counter-arguments to the high VHE luminosities of five of these objects. (i) The uncertainties in a background model are suggestive of a lower flux of CTB 37A (which are illustrated in Fig. 5 of Abdalla et al. (2018a)) than that published in Aharonian et al. (2008b), (ii) the estimated energies of the relativistic particles are too high for the hadronic and leptonic scenarios applied to CTB 37B, suggesting that the distance of $13.2 \mathrm{kpc}$ to this source is an overestimate (Xin et al. 2016), (iii) SNR G359.1-0.5 is likely only to be responsible for a part of the VHE gamma-ray source, HESS J1745-303, given that the solid angle of region A (Aharonian et al. 2008a) that embeds a molecular cloud, as viewed from the center of G359.10.5 , is about an order of magnitude larger than the angles of the other emitting regions (also Hayakawa et al.2012), but the contribution from region A to the total flux of HESS J1745-303 is subdominant (Aharonian et al.2008a), (iv) the distance to SNR $\mathrm{G} 106.3+2.7$ is uncertain, and if a distance of $0.8 \mathrm{kpc}$ instead of $12 \mathrm{kpc}$ is adopted, then its estimated VHE luminosity decreases significantly (Acciari et al. 2009), (v) HESS 1804-216 is likely to be dominated by a PWN surrounding the pulsar PSR B1800-21, but not by SNR G8.7-0.1, as demonstrated through the Fermi-LAT spectral and spatial analyses (Liu et al. 2019) and on the basis of a comparison of its properties with those of the established VHE PWN (Abdalla et al.2018b). Finally, SNR G318.2+0.1 is associated with the VHE source HESS J1457-593 and is suggested to be originated from an interaction between $\mathrm{G} 318.2+0.1$ and a molecular cloud seen in ${ }^{12} \mathrm{CO}$ data (Hofverberg et al. 2010b), and it therefore cannot be directly compared to SNR N132D. It can also be noted here that in the case of the composite SNR G338.3-0.0 associated with HESS J1640465 , the scenario in which the VHE emission originates predominantly from the PWN of the subsequently discovered PSR $\mathrm{J} 1640-4631$ is highly rated in the context of the VHE PWN population (Abdalla et al. 2018b); it remains plausible nonetheless that a significant part of the gamma-ray emission from HESS J1640-465 originates in the SNR shell (Abramowski et al. 2014).

\section{References}

Abdalla, H., Abramowski, A., Aharonian, F., et al. 2018b, A\&A, 612, A2 Abdalla, H., Abramowski, A., Aharonian, F., et al. 2018e, A\&A, 612, A7 Abdalla, H., Abramowski, A., Aharonian, F., et al. 2018a, A\&A, 612, A1 Abdalla, H., Abramowski, A., Aharonian, F., et al. 2018e, A \& A, 612 Abdalla, H., Abramowski, A., Aharonian, F., et al. 2018d, A \& A, 612, A6 Abdalla, H., Abramowski, A., Aharonian, F., et al. 2018f, A\&A, 610 Abdalla, H., Abramowski, A., Aharonian, F., et al. 2018c, A\&A, 612, A3 Abdollahi, S., Acero, F., Ackermann, M., et al. 2020, ApJS, 247, 33

Abeysekara, A. U., Archer, A., Benbow, W., et al. 2020, ApJ, 894, 51 Abramowski, Acero, F., Aharonian, F., et al. 2012, A\&A, 545, L2 Abramowski, A., Acero, F., Aharonian, F., et al. 2012, A\&A, 545, L2, publisher: EDP Sciences

Abramowski, A., Aharonian, F., Ait Benkhali, F., et al. 2008, A\&A, 481, 401 Abramowski, A., Aharonian, F., Ait Benkhali, F., et al. 2015a, A\&A, 575, A81 Abramowski, A., Aharonian, F., Benkhali, F. A., et al. 2014, MNRAS, 439, 2828 Abramowski, A., Aharonian, F., Benkhali, F. A., et al. 2015b, Science, 347, 406 Abramowski, A. Ostrowski, M., Oya, I., Panter, M., \& Parsons. 2014, MNRAS, 441,3640

Acciari, V. A., Aliu, E., Arlen, T., et al. 2009, ApJ, 703, L6

Acciari, V. A., Ansoldi, S., Antonelli, L. A., et al. 2020, A \& A, 635, A158

Acero, F., Ackermann, M., Ajello, M., et al. 2016, ApJ Supplement Series, 224, 8

Acharya, B. S., Agudo, I., Al Samarai, I., et al. 2019, Science with the Cherenkov Telescope Array

Ackermann, M., Ajello, M., Allafort, A., et al. 2013, Science, 339, 807

Ackermann, M., Albert, A., Atwood, W. B., et al. 2016, A \& A, 586, A71

Aharonian, F., Ait Benkhali, F., Akhperjanian, A. G., et al. 2008, A\&A, 481, 401

Aharonian, F., Akhperjanian, A., Barrio, A., et al. 2001, A\&A, 370, 112

Aharonian, F., Akhperjanian, A. G., Barres de Almeida, U., et al. 2008a, A\&A, 483, 509

Aharonian, F., Akhperjanian, A. G., Barres de Almeida, U., et al. 2008b, A\&A, 490,685

Aharonian, F., Akhperjanian, A. G., Bazer-Bachi, A. R., et al. 2006, A\&A, 457, 899

Aharonian, F. A. \& Atoyan, A. M. 1996, A\&A, 309, 917

Aharonian, F. A., Kelner, S. R., \& Prosekin, A. Y. 2010, Physical Review D, 82, 043002

Ahnen, M. L., Ansoldi, S., Antonelli, L. A., et al. 2017, MNRAS, 472, 2956

Akaike, H. 1974, IEEE transactions on automatic control, 19, 716

Asvarov, A. I., Guseinov, O. H., Kasumov, F. K., \& Dogel', V. A. 1990, A\&A, 229,196

Atwood, W. B., Abdo, A. A., Ackermann, M., et al. 2009, ApJ, 697, 1071

Bamba, A., Ohira, Y., Yamazaki, R., et al. 2018, ApJ, 854

Bamba, A., Yamazaki, R., Yoshida, T., Terasawa, T., \& Koyama, K. 2005, The Astrophysical Journal, 621, 793

Banas, K. R., Hughes, J. P., Bronfman, L., \& Nyman, L.-A. 1997, ApJ, 480, 607 Baring, M. G., Ellison, D. C., Reynolds, S. P., Grenier, I. A., \& Goret, P. 1999, ApJ, 513, 311

Behar, E., Rasmussen, A. P., Griffiths, R. G., et al. 2001, A\&A, 365, L242

Berezhko, E. G. 1996, Astroparticle Physics, 5, 367

Berge, D., Funk, S., \& Hinton, J. 2007, A\&A, 466, 1219

Borkowski, K. J., Hendrick, S. P., \& Reynolds, S. P. 2007, ApJ, 671, L45

Braiding, C., Wong, G. F., Maxted, N. I., et al. 2018, Publications of the Astronomical Society of Australia, 35, e029

Burbidge, G. R. 1956, ApJ, 124, 416

Campana, R., Massaro, E., \& Bernieri, E. 2018, Ap\&SS, 363, 144 Cardillo, M., Tavani, M., Giuliani, A., et al. 2014, A \& A, 565, A74

Cash, W. 1979, ApJ, 228, 939

Chen, Y., Zhang, F., Williams, R. M., \& Wang, Q. D. 2003, ApJ, 595, 227 Cristofari, P., Blasi, P., \& Amato, E. 2020, Astroparticle Physics, 123, 102492 de Naurois, M. \& Rolland, L. 2009, Astroparticle Physics, 32, 231 Dermer, C. D. \& Powale, G. 2013, A\&A, 553, A34

Dickel, J. R. \& Milne, D. K. 1995, The Astronomical Journal, 109, 200 Fernandez, D., Hahn, J., Marandon, V., et al. 2013, in International Cosmic Ray Conference, Vol. 33, International Cosmic Ray Conference, 2682

France, K., Beasley, M., Keeney, B. A., et al. 2009, ApJ, 707, L27

Freeman, P., Doe, S., \& Siemiginowska, A. 2001, in Astronomical Data Analysis, ed. J.-L. Starck \& F. D. Murtagh, Vol. 4477, International Society for Optics and Photonics (SPIE), $76-87$

Gabici, S. 2017, in American Institute of Physics Conference Series, Vol. 1792, 6th International Symposium on High Energy Gamma-Ray Astronomy, 020002

Gabici, S. \& Aharonian, F. A. 2007, ApJ, 665, L131

Ginzburg, V. L. 1972, Nature Physical Science, 239, 8 
Ginzburg, V. L. \& Syrovatskii, S. I. 1964, The Origin of Cosmic Rays (Oxford, New York, Pergamon Press)

Hahn, J., De los Reyes, R., Bernlöhr, K., et al. 2014, Astroparticle Physics, 54, 25

Hayakawa, T., Torii, K., Enokiya, R., Amano, T., \& Fukui, Y. 2012, PASJ, 64, 8 Helder, E. A., Vink, J., Bykov, A. M., et al. 2012, Space Sci. Rev., 173, 369

Hofverberg, P., Chaves, R. C. G., Fiasson, A., et al. 2010a, in 25th Texas Symposium on Relativistic Astrophysics, 196

Hofverberg, P., Chaves, R. C. G., Fiasson, A., et al. 2010b, in 25th Texas Symposium on Relativistic Astrophysics, 196

Hughes, J. P. 1987, ApJ, 314, 103

Hughes, J. P., Hayashi, I., \& Koyama, K. 1998, ApJ, 505, 732

Hwang, U., Petre, R., \& Flanagan, K. A. 2008, ApJ, 676, 378

Kafexhiu, E., Aharonian, F., Taylor, A. M., \& Vila, G. S. 2014, Physical Review D, 90, 123014

Katz, B. \& Waxman, E. 2008, J. Cosmology Astropart. Phys., 2008, 018

Khangulyan, D., Aharonian, F. A., \& Kelner, S. R. 2014, ApJ, 783, 100

Lagage, P. O. \& Cesarsky, C. J. 1983, A\&A, 125, 249

Lemoine-Goumard, M., Grondin, M.-H., Acero, F., et al. 2014, ApJ, 794

Li, T.-P. \& Ma, Y.-Q. 1983, ApJ, 272, 317

Liu, B., Yang, R.-z., Sun, X.-n., Aharonian, F., \& Chen, Y. 2019, ApJ, 881, 94 Longair, M. S. 2011, High Energy Astrophysics (University of Cambridge)

Maggi, P., Haberl, F., Kavanagh, P. J., et al. 2016, A\&A, 585, A162

Makino, K., Fujita, Y., Nobukawa, K. K., Matsumoto, H., \& Ohira, Y. 2019, PASJ, 71,78

Malkov, M. A. \& Drury, L. O. 2001, Reports on Progress in Physics, 64, 429

Mathewson, D. S., Ford, V. L., Dopita, M. A., et al. 1983, ApJ Supplement Series, 51,345

Moré, J. J. 1978, in Numerical analysis (Springer), 105-116

Moskalenko, I. V., Porter, T. A., Malkov, M. A., \& Diamond, P. H. 2008, in International Cosmic Ray Conference, Vol. 2, International Cosmic Ray Conference, 763-766

Ohm, S., van Eldik, C., \& Egberts, K. 2009, Astroparticle Physics, 31, 383

Parsons, R. D. \& Hinton, J. A. 2014, Astroparticle Physics, 56, 26

Pietrzyński, G., Graczyk, D., Gallenne, A., et al. 2019, Nature, 567, 200

Reed, J. E., Hester, J. J., Fabian, A. C., \& Winkler, P. F. 1995, ApJ, 440, 706

Sano, H., Fujii, K., Yamane, Y., et al. 2017, in American Institute of Physics Conference Series, Vol. 1792, 6th International Symposium on High Energy Gamma-Ray Astronomy, 040038

Sano, H., Plucinsky, P. P., Bamba, A., et al. 2020, ApJ, 902, 53

Seok, J. Y., Koo, B.-C., \& Onaka, T. 2013, ApJ, 779, 134

Sharda, P., Gaetz, T., Kashyap, V., \& Plucinsky, P. 2020, arXiv e-prints, arXiv:2004.07366

Slane, P., Castro, D., Funk, S., et al. 2010, ApJ, 720, 266

Spengler, G. 2020, A\&A, 633, A138

Suzuki, H., Bamba, A., Yamazaki, R., \& Ohira, Y. 2020, PASJ, 72, 72

Tappe, A., Rho, J., Boersma, C., \& Micelotta, E. R. 2012, ApJ, 754, 132

Tappe, A., Rho, J., \& Reach, W. T. 2007, ApJ, 659, 1794

Thorstensen, J. R., Fesen, R. A., \& van den Bergh, S. 2001, AJ, 122, 297

Tsuji, N. \& Uchiyama, Y. 2016, PASJ, 68, 108

Vink, J. 2008, A\&A, 486, 837

Vink, J. 2020, Physics and Evolution of Supernova Remnants

Vogt, F. \& Dopita, M. A. 2011, Astrophysics and Space Science, 331, 521-535

Völk, H. J. \& Biermann, P. L. 1988, ApJ, 333, L65

Weaver, R., McCray, R., Castor, J., Shapiro, P., \& Moore, R. 1977, ApJ, 218, 377

Williams, B. J., Borkowski, K. J., Reynolds, S. P., et al. 2006, ApJ, 652, L33

Wong, T., Hughes, A., Ott, J., et al. 2011, ApJS, 197, 16

Xin, Y.-L., Guo, X.-L., Liao, N.-H., et al. 2017, ApJ, 843, 90

Xin, Y.-L., Liang, Y.-F., Li, X., et al. 2016, ApJ, 817, 64

Young, P. A., Fryer, C. L., Hungerford, A., et al. 2006, ApJ, 640, 891

Zabalza, V. 2015, Proc. of International Cosmic Ray Conference 2015, 922

Zeng, H., Xin, Y., \& Liu, S. 2019, ApJ, 874, 50
1 University of Namibia, Department of Physics, Private Bag 13301, Windhoek 10005, Namibia

2 Dublin Institute for Advanced Studies, 31 Fitzwilliam Place, Dublin 2, Ireland

3 Max-Planck-Institut für Kernphysik, P.O. Box 103980, D 69029 Heidelberg, Germany

${ }^{4}$ High Energy Astrophysics Laboratory, RAU, 123 Hovsep Emin St Yerevan 0051, Armenia

5 Aix Marseille Université, CNRS/IN2P3, CPPM, Marseille, France

${ }^{6}$ Centre for Space Research, North-West University, Potchefstroom 2520, South Africa

7 Laboratoire d'Annecy de Physique des Particules, Univ. Grenoble Alpes, Univ. Savoie Mont Blanc, CNRS, LAPP, 74000 Annecy, France

${ }^{8}$ University of Oxford, Department of Physics, Denys Wilkinson Building, Keble Road, Oxford OX1 3RH, UK

${ }^{9}$ IRFU, CEA, Université Paris-Saclay, F-91191 Gif-sur-Yvette, France

${ }^{10}$ Instytut Fizyki Jạdrowej PAN, ul. Radzikowskiego 152, 31-342 Kraków, Poland

11 DESY, D-15738 Zeuthen, Germany

12 Obserwatorium Astronomiczne, Uniwersytet Jagielloński, ul. Orla 171, 30-244 Kraków, Poland

13 School of Physics, University of the Witwatersrand, 1 Jan Smuts Avenue, Braamfontein, Johannesburg, 2050 South Africa

14 Department of Physics and Electrical Engineering, Linnaeus University, 35195 Växjö, Sweden

15 Institut für Astronomie und Astrophysik, Universität Tübingen, Sand 1, D 72076 Tübingen, Germany

16 Laboratoire Univers et Théories, Observatoire de Paris, Université PSL, CNRS, Université de Paris, 92190 Meudon, France

17 Sorbonne Université, Université Paris Diderot, Sorbonne Paris Cité, CNRS/IN2P3, Laboratoire de Physique Nucléaire et de Hautes Energies, LPNHE, 4 Place Jussieu, F-75252 Paris, France

18 Astronomical Observatory, The University of Warsaw, Al. Ujazdowskie 4, 00-478 Warsaw, Poland

19 Friedrich-Alexander-Universität Erlangen-Nürnberg, Erlangen Centre for Astroparticle Physics, Erwin-Rommel-Str. 1, D 91058 Erlangen, Germany

20 Université Bordeaux, CNRS/IN2P3, Centre d'Études Nucléaires de Bordeaux Gradignan, 33175 Gradignan, France

${ }^{21}$ Université de Paris, CNRS, Astroparticule et Cosmologie, F-75013 Paris, France

22 Department of Physics and Astronomy, The University of Leicester, University Road, Leicester, LE1 7RH, United Kingdom

23 Institut für Physik und Astronomie, Universität Potsdam, KarlLiebknecht-Strasse 24/25, D 14476 Potsdam, Germany

24 School of Physical Sciences, University of Adelaide, Adelaide 5005, Australia

25 Laboratoire Leprince-Ringuet, École Polytechnique, CNRS, Institut Polytechnique de Paris, F-91128 Palaiseau, France

26 Laboratoire Univers et Particules de Montpellier, Université Montpellier, CNRS/IN2P3, CC 72, Place Eugène Bataillon, F-34095 Montpellier Cedex 5, France

27 Institut für Astro- und Teilchenphysik, Leopold-FranzensUniversität Innsbruck, A-6020 Innsbruck, Austria

28 Universität Hamburg, Institut für Experimentalphysik, Luruper Chaussee 149, D 22761 Hamburg, Germany

29 Landessternwarte, Universität Heidelberg, Königstuhl, D 69117 Heidelberg, Germany

${ }^{30}$ Institute of Astronomy, Faculty of Physics, Astronomy and Informatics, Nicolaus Copernicus University, Grudziadzka 5, 87-100 Torun, Poland

31 Department of Physics, Rikkyo University, 3-34-1 Nishi-Ikebukuro, Toshima-ku, Tokyo 171-8501, Japan

32 Nicolaus Copernicus Astronomical Center, Polish Academy of Sciences, ul. Bartycka 18, 00-716 Warsaw, Poland

33 Institut für Physik, Humboldt-Universität zu Berlin, Newtonstr. 15, D 12489 Berlin, Germany

34 Department of Physics, University of the Free State, PO Box 339, Bloemfontein 9300, South Africa Article number, page 13 of 13

35 GRAPPA, Anton Pannekoek Institute for Astronomy, University of Amsterdam, Science Park 904, 1098 XH Amsterdam, The Netherlands

36 Yerevan Physics Institute, 2 Alikhanian Brothers St., 375036 Yerevan, Armenia 